\title{
CHANDRA REVEALS TWIN X-RAY JETS IN THE POWERFUL FR II RADIO GALAXY 3C 353
}

\author{
J. KATAOKA ${ }^{1}$, Ł. STAWARZ $^{2,3}$, D.E. HARRIS ${ }^{4}$, A. SIEMIGINOWSKA ${ }^{4}$, M. OSTROWSKI ${ }^{3}$, \\ M.R. SWAIN ${ }^{5}$, M.J. HARdCASTLE, J.L. GoOdGER ${ }^{6}$, K. IWASAWA ${ }^{7}$, AND P.G. EdWARds ${ }^{8}$ \\ Accepted version June 7, 2008
}

\begin{abstract}
We report X-ray imaging of the powerful FR II radio galaxy 3C 353 using the Chandra X-ray Observatory. 3C 353's two 4"-wide and 2'-long jets allow us to study in detail the internal structure of the large-scale relativistic outflows at both radio and X-ray photon energies with the sub-arcsecond spatial resolution provided by the VLA and Chandra instruments. In a $90 \mathrm{ks}$ Chandra observation, we have detected X-ray emission from most radio structures in $3 \mathrm{C} 353$, including the nucleus, the jet and the counterjet, the terminal jet regions (hotspots), and one radio lobe. We show that the detection of the X-ray emission associated with the radio knots and counterknots, which is most likely non-thermal in origin, puts several crucial constraints on the X-ray emission mechanisms in powerful large-scale jets of quasars and FR II sources. In particular, we show that this detection is inconsistent with the inverse-Compton model proposed in the literature, and instead implies a synchrotron origin of the X-ray jet photons. We also find that the width of the X-ray counterjet is possibly narrower than that measured in radio bands, that the radio-to-X-ray flux ratio decreases systematically downstream along the jets, and that there are substantial (kpc-scale) offsets between the positions of the X-ray and radio intensity maxima within each knot, whose magnitudes increase away from the nucleus. We discuss all these findings in the wider context of the physics of extragalactic jets, proposing some particular though not definitive solutions or interpretations for each problem. In general, we find that the synchrotron X-ray emission of extragalactic large-scale jets is not only shaped by the global hydrodynamical configuration of the outflows, but is also likely to be very sensitive to the microscopic parameters of the jet plasma. A complete, self-consistent model for the X-ray emission of extragalactic jets still remains elusive.
\end{abstract}

Subject headings: galaxies: active — galaxies: jets — galaxies: individual (3C 353) — radiation mechanisms: nonthermal - X-rays: general

\section{INTRODUCTION}

Extragalactic jets constitute the longest collimated structures in the Universe. They transport huge amounts of energy from the nuclei of active galaxies out to kpc or Mpc distances, significantly affecting the properties of the surrounding intracluster/intergalactic medium. Jets have been extensively studied in the radio domain on different scales since the very beginning of the development of modern radio interferometers (Bridle \& Perley 1984, Begelman et al. 1984). More recently, the excellent spatial resolution of the Chandra X-ray Observatory (and, to a lesser extent, of other X-ray satellites like $X M M$-Newton) has allowed us to image large-scale structures in powerful extragalactic radio sources at X-ray frequencies as well, and thus has opened a new era in studying the high energy emission of these objects. More than 100 radio-loud AGNs are now known to possess X-ray counterparts to their radio jets, hotspots or lobes on kpc-to-Mpc scales (e.g., Harris

\footnotetext{
Electronic address: kataoka@hp.phys.titech.ac.jp

${ }^{1}$ Department of Physics, Tokyo Institute of Technology, 2-12-1 Ohokayama, Meguro, Tokyo, 152-8551, Japan

${ }^{2}$ Kavli Institute for Particle Astrophysics and Cosmology, Stanford University, Stanford, CA 94305, USA

${ }^{3}$ Obserwatorium Astronomiczne, Uniwersytet Jagielloński, ul. Orla 171, 30-244 Kraków, Poland

${ }^{4}$ Harvard-Smithsonian Center for Astrophysics, 60 Garden St., Cambridge, MA 02138

5 Jet Propulsion Laboratory, California Institute of Technology, 4800 Oak Grove Drive, Pasadena, CA 91109

${ }^{6}$ University of Hertfordshire, College Lane, Hatfield, Hertfordshire AL 10 $9 \mathrm{AB}, \mathrm{UK}$

7 INAF-Osservatorio Astronomico di Bologna, Via Ranzani, 1, 40127, Bologna, Italy

${ }^{8}$ Australia Telescope National Facility, CSIRO, Locked Bag 194, Narrabri NSW 2390, Australia
}

\& Krawczynski 2002; 2006, Stawarz 2003, Hardcastle et al. 2004, Sambruna et al. 2004, Kataoka \& Stawarz 2005, Marshall et al. 2005a, Croston et al. 2005, Tavecchio et al. 2005, Hardcastle 2006, and references therein). The X-ray emission observed from the extended lobes is well understood and modeled in terms of the inverse Comptonization of the cosmic microwave background photons by the low-energy electrons (IC/CMB; see the discussion in Kataoka \& Stawarz 2005, and Croston et al. 2005), providing strong evidence for approximate energy equipartition between the radiating electrons and the lobe magnetic field (with the particle pressure dominating over the magnetic pressure by up to one order of magnitude). However, the origin of the $0.1-10 \mathrm{keV}$ radiation detected from the large-scale jets and hotspots is still widely debated and, to some extent, controversial.

The X-ray emission of the terminal regions (the hotspots) of powerful jets is consistent with the synchrotron self-Compton (SSC) emission model, in which radio-emitting electrons accelerated at the terminal shock inverse-Compton-scatter synchrotron radio photons to $\mathrm{keV}$ energies (e.g., Hardcastle et al. 2004, Kataoka \& Stawarz 2005, and references therein). Modeling of X-rays from this emission process allows us to extract several crucial jet parameters. In particular, the observations imply rough equipartition of the energy densities stored in radiating electrons, $U_{\mathrm{e}}$, and magnetic field, $U_{\mathrm{B}} \lesssim U_{\mathrm{e}}$, and it has been argued that they indicate a dynamical role for non-relativistic protons in the outflow (see Stawarz et al. 2007 for the case of the radio galaxy Cygnus A). On the other hand, the X-ray emission of the hotspots in low-power jets is in disagreement with the predictions of the simple SSC model (see the discussion in Hardcastle et al. 2004). The complex X-ray morphology and spectral character 
of the broad-band hotspot emission in objects like 3C 227 or 3C 327 suggest instead that we are seeing synchrotron emission from high-energy electrons accelerated continuously in the extended and turbulent jet termination regions (Hardcastle et al. 2007a, Fan et al. 2008). The origin of the bright X-ray knots within the jets themselves (hereafter 'jet knots') is also a matter of a debate. In nearby, low-power FR I sources (like M 87 and Centaurus A), the typical radio-to-X-ray spectra of the jet knots are consistent with a single smoothly broken power-law continuum, indicating synchrotron radiation from a single electron population extending up to the highest energies $\left(\gamma \equiv E_{\mathrm{e}} / m_{\mathrm{e}} c^{2} \sim 10^{8}\right.$; e.g., Marshall et al. 2002, Hardcastle et al. 2003). Yet the particular acceleration processes involved, the multi-component character of the high-energy emission, and the main factors determining the spectral shape of the broad-band jet emission, are far from being understood (see the discussions in, e.g., Kataoka et al. 2006, Laing et al. 2006a, Honda \& Honda 2007).

The most controversial issue, however, is the origin of the intense X-ray emission detected from knots in powerful quasar jets, such as PKS 0637-752 or 3C 273 (Schwartz et al. 2000, Marshall et al. 2001, respectively). Here the X-ray knot spectra are much brighter than expected from a simple extrapolation of the radio-to-optical synchrotron continua, indicating that an additional or separate spectral component dominates the jet's radiative output at high (X-ray) photon energies. Very often, this emission is modeled in terms of inverse-Comptonization of the CMB photon field by low-energy $\left(\gamma \lesssim 10^{3}\right.$ ) electrons (Tavecchio et al. 2000, Celotti et al. 2001), which typically requires highly relativistic jet bulk velocities on kpc-Mpc scales in order to maintain rough equipartition between electrons and magnetic field and to fulfill the minimum power condition. The derived jet bulk Lorentz factors $\Gamma_{\text {jet }} \gtrsim 10$ are comparable to those inferred for the pc-scale jets; (Harris \& Krawczynski 2002, Sambruna et al. 2004, Marshall et al. 2005a). Unfortunately, the global dynamics of large-scale jets in powerful sources are largely unknown, and different arguments in favor of and against highly relativistic bulk velocities on kpc-Mpc scales (obtained by means of the analysis of the radio or optical jet emission; e.g., Wardle \& Aaron 1997, Hardcastle et al. 1999, Scarpa \& Urry 2002), are not conclusive. Thus, the IC/CMB model may be considered to be strong support for the idea that these outflows do indeed propagate from sub-pc scales with little energy dissipation, efficiently transporting energy in the form of bulk kinetic motion, and depositing it far away from the active nuclei (Tavecchio et al. 2004; 2007, Sambruna et al. 2006; but see the discussion in Hardcastle 2006). This important conclusion is often claimed to be consistent with the observed one-sideness of the extragalactic X-ray jets detected by Chandra, since jet one-sideness is a simple and natural consequence of relativistic beaming.

On the other hand, if there is significant beaming in powerful jets on large scales, then the detection of bright X-ray jet emission from FR II radio galaxies, which are believed to be analogous systems to radio loud quasars but to be viewed with the jets at large angles to the line of sight, should be considered as unlikely. Such emission has, however, been detected in several objects (e.g., 3C 303, 3C 15, Pictor A, or 3C 403; see Kataoka et al. 2003a,b, Hardcastle \& Croston 2005, and Kraft et al. 2005, respectively). Obviously, detection of any X-ray counterjet would be of primary importance in this respect, since it would automatically exclude significant beaming, and thus impose very severe constraints on the jet emission models. In FR I sources, where the emission mechanism is generally supposed to be synchrotron, possible or likely detections of X-ray counterjets have been reported in 3C 270 and Centaurus A (Chiaberge et al. 2003, Hardcastle et al. 2007b, respectively). In the latter case the counterjet detection is almost certain, since extended $\mathrm{X}$-ray emission coincident with the extended radio features in the receding jet rules out a claim that all of the observed X-ray counterparts to the radio counter-knots may be due to chance coincidence of the jet-related radio features with X-ray binaries of the Centaurus A host galaxy. However, in more powerful sources, there has been no definitive counterjet detection to date. Possible counterjets have been reported in the intermediate FR I/FR II object 4C 29.30 (Sambruna et al. 2004), as well as in the broad-line FR II radio galaxy Pictor A (Hardcastle \& Croston 2005). In these cases, it is unclear whether the X-ray emission is non-thermal in nature, because with the available very limited photon statistics any detailed spectral analysis is impossible, and in addition it is rather difficult to claim one-to-one morphological correspondence between the $\mathrm{X}$-ray and radio structures (especially since the counterjet in the prototype FR II source Pictor A is not detected at radio frequencies). In X-ray maps of the classical double (narrowline FR II radio galaxy) Cygnus A, linear features aligned - but not co-spatial - with the radio jet and the counterjet have been noted, but their direct connection with the radio jet plasma is extremely vague (Smith et al. 2002, Steenbrugge et al. 2008). It is then possible that in this, and some other possible X-ray counterjet sources, the aligned X-ray structures on the counterjet side are due to thermal radiation of shocked galactic/intergalactic gas interacting with the jet plasma, as observed in the distant ( $z=2.2)$ radio galaxy PKS 1138-262 (Carilli et al. 2002). We note in this context that Cygnus $\mathrm{A}$ is located in the very center of a rich cluster environment, while the restarting 4C 29.30 radio galaxy is known to have clear signatures of the interaction between the radio jets and the ambient line-emitting gas (van Breugel et al. 1986, Jamrozy et al. 2007). A clear spatial association between the radio and $\mathrm{X}$-ray jets and the capability to distinguish non-thermal and thermal emission are the key requirements for a convincing counterjet detection in a powerful source.

An alternative to the inverse-Compton model as an interpretation of the X-ray emission of powerful jets involves synchrotron emission from high-energy electrons, which, in order to be consistent with the constraints on optical emission, must often either be characterized by a 'non-standard' (concave) energy distribution, or must constitute a separate population to the ones emitting the radio-to-optical continuum. It has been noted that such an electron population can arise due to the continuous and efficient stochastic acceleration processes expected to take place within the extended jet volumes (Stawarz et al. 2004), and will be seen preferentially within turbulent jet boundary layers with significant velocity shear (Stawarz \& Ostrowski 2002). A very strong support for the synchrotron hypothesis was recently provided by detailed multiwavelength observations of the jet in the quasar 3C 273 (Jester et al. 2006, 2007, Uchiyama et al. 2006). The observations showed in particular that the X-ray spectra are significantly softer than the radio spectra in most regions of the 3C 273 outflow, and that they are compatible with extrapolating the X-ray power law down to the polarized (and therefore synchrotron in origin) UV/optical continuum. These findings are in strong disagreement with the predictions of the IC/CMB model. In addition, a detailed analysis of the 
broad-band emission of several other quasar jets supports the synchrotron hypothesis, pointing out an important role of the jet velocity structure (consisting of a fast spine and a slower boundary layer/outer sheath) in shaping the jet high-energy radiation (e.g., Hardcastle 2006, Jester et al. 2006, Siemiginowska et al. 2007). Such jet velocity structure is in fact always seen in numerical modeling of the evolution and propagation of extragalactic relativistic jets (e.g., Aloy et al. 1999, Leismann et al. 2005, Mizuno et al. 2007), since it results inevitably from the non-linear growth of Kelvin-Helmholtz instabilities on the jet surface (see the recent studies by Perucho et al. 2007, Hardee 2007, Meliani \& Keppens 2007, and references therein). It is not yet established, however, if the jet boundary layers are indeed the places of the enhanced acceleration of high-energy particles. Nor is the exact velocity profile at the jet boundaries precisely known; in fact, there are reasons to believe it may be far from the widely expected monotonic one, and may even exhibit a sharp increase in the bulk velocity of the outflow at the jet edges (Aloy \& Rezzolla 2006, Mizuno et al. 2008). The radiative signatures of such 'anomalous' shear layers, in the context of the high-energy emission from extragalactic large-scale jets, were recently investigated by Aloy \& Mimica (2008).

Observational studies of the velocity structure of extragalactic jets and its relationship to their broad-band emission are hampered by the difficulties in resolving the outflows transversely, especially at high energies. Detailed radio studies and sophisticated modeling of the polarization and total intensity in the radio of several nearby FR I sources (like 3C 31, NGC 315, or 3C 296; Laing \& Bridle 2004, Laing et al. 2006a,b, respectively) do reveal jet radial velocity structures, and indicate that the flatter-spectrum radio regions are indeed often associated with the jet boundary shear layers. Unfortunately, only a few kpc-scale low-power jets, e.g., the one hosted by the radio galaxy Centaurus $\mathrm{A}$, can be resolved at X-ray frequencies by the Chandra instrument. The Cen A observations show interestingly limb-brightened X-ray morphology, but there is no obvious relation with the spectral shape of the synchrotron X-ray continuum (Hardcastle et al. 2003, 2007b, Kataoka et al. 2006, Worrall et al. 2008). In particular, the diffuse component of the Centaurus A X-ray jet is characterized by a constant spectral index across the jet (Kataoka et al. 2006), while the X-ray spectra of isolated knots seem to be steeper within the jet sheath when compared to the X-ray spectra of the knots within the spine (Worrall et al. 2008). Recently, two 'intermediate' FR I/FR II jets in the BL Lacertae objects 3C 371 and PKS 2201+44 were also resolved transversely in X-rays (Sambruna et al. 2007). The observations indicated a synchrotron origin of the detected $\mathrm{keV}$ photons from all the jet components, and suggested slightly steeper X-ray spectra of the jet edges when compared with the jet spines. Interestingly, the width of the X-ray jets in these two sources is not smaller than the widths of their optical and radio counterparts. Needless to say, no quasar or powerful FR II jet has been resolved till now at X-ray frequencies. This is true even for the particularly bright and wide 3C 273 jet, which, although imaged in detail at optical and radio frequencies (Jester et al. 2005), cannot be resolved transversely at higher photon energies. For this source, however, the limits to the width of the X-ray jet are smaller than the sized measured in the optical and radio bands at the position of the bright knots (Jester et al. 2006), but not necessarily in the interknot regions (see Marshall et al. 2005b).

The FR II radio galaxy 3C 353 provides one of the best-

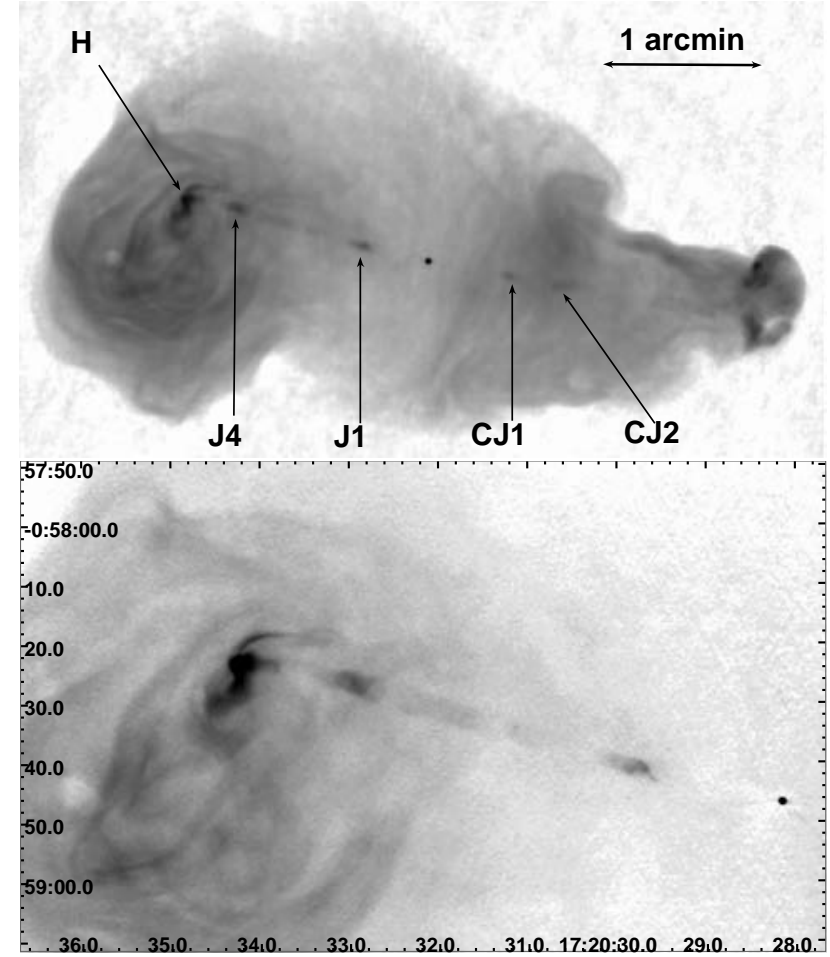

FIG. 1.- Above: $1.4 \mathrm{GHz}$ VLA image of $3 \mathrm{C} 353$ at $1.3^{\prime \prime}$ resolution (adapted from Swain et al. 1998). Below: a zoom in on the eastern jet $\left(0.44^{\prime \prime}\right.$ resolution at $\left.8.4 \mathrm{GHz}\right)$ shows that the jet is well resolved, and clearly extended compared to the unresolved nucleus.

known examples of FR II jets that can be resolved at both radio and X-ray wavelengths. $3 \mathrm{C} 353(z=0.0304)$ is the fourth strongest radio source in the $3 \mathrm{C}$ catalog, with a total flux density $S_{\nu} \approx 57 \mathrm{Jy}$ at $1.4 \mathrm{GHz}$, and a projected size $\sim 4.5^{\prime}$. It exhibits hotspots and a pair of large-scale FR IItype jets, clearly visible within filamentary lobes (Swain et al. 1998). The jets in 3C 353, constituting about $1 \%$ of the entire source luminosity, are well collimated trains of knots with an average width $\approx 4^{\prime \prime}$ and jet-counterjet radio brightness asymmetry $\approx 2$ (Swain 1996). Both total and polarized intensity profiles across the jets indicate that the bulk of the jet radio emission is produced at the jet edges, and so presumably within a boundary shear layer (Swain 1996, Swain et al. 1998). Since these jets are among only a very few FR II jets wide enough to be resolved in X-rays, we planned and conducted a deep Chandra observation of 3C 353, in order to investigate the multiwavelength structure of powerful FR II outflows. We report on the results of these observations in the present paper.

A complication in X-ray studies of $3 \mathrm{C} 353$ is the X-ray emission from its large-scale environment. 3C 353 is situated at the edge of the cluster $\mathrm{Zw} 1718.1-0108$, which is a complex and dynamic system captured at the moment of an on-going merger (Iwasawa et al. 2000). Based on a $40 \mathrm{ks} A S C A$ observation, Iwasawa et al. reported that this cluster is characterized by a relatively low X-ray luminosity, $\sim 5 \times 10^{43} \mathrm{erg} \mathrm{s}^{-1}$, high temperature, $k T=3-5 \mathrm{keV}$, and disturbed morphology, extended over $\sim 30^{\prime}$. The small field of view of Chandra, together with the roll angle of Chandra during our observations, prevent us from carrying out detailed studies of the interaction between the radio source and the cluster. Therefore, in this paper we focus solely on the analysis of the non-thermal emission from the nucleus, jets, hotspots and lobes. Analysis 
TABLE 1

X-RAY SOURCES DETECTED WITH WAVEDETECT ALONG THE 3C 353 JET.

\begin{tabular}{lllcccc}
\hline $\begin{array}{l}\text { X-ray } \\
\text { jet region }\end{array}$ & $\begin{array}{l}\text { associated } \\
\text { X-ray features }\end{array}$ & $\begin{array}{l}\text { nearby } \\
\text { radio knot }\end{array}$ & ${\text { RA }(J 2000)^{a}}^{a}$ & DEC $(J 2000)^{a}$ & Net counts $^{b}$ & Significance $(\sigma)^{d}$ \\
\hline EJ1 & E21/E23 & J1 & 172029.7 & -005841.6 & $46.9 \pm 7.2$ & 13.7 \\
EJ2 & E70/E73 & J4 & 172032.8 & -005826.6 & $12.0 \pm 4.0$ & 3.8 \\
EJ3 & E88 & H & 172033.8 & -005823.7 & $32.2 \pm 6.3$ & 8.2 \\
WJ1 & W47 & CJ2 & 172025.0 & -005855.1 & $32.0 \pm 6.0$ & 10.0 \\
WJ2 & W120a/b & $\ldots$ & 172020.3 & -005909.9 & $12.3 \pm 3.7$ & 4.8 \\
\hline
\end{tabular}

Note: all the errors are $1 \sigma$.

${ }^{\text {a }}$ Coordinate center of the detected X-ray sources.

${ }^{b}$ Net photon counts given by the WAVDETECT source detection algorithm.

${ }^{\mathrm{c}}$ Statistical significance of detected jet features.

of the X-ray emission from the cluster and lobes based on recent XMM-Newton observations is presented in Goodger et al. (2008: hereafter G08); they found slightly different temperatures for the southern and northern parts of the cluster, which supported the idea that these two components were originally separate features that are now undergoing a smooth merger, and also showed that the X-ray emission detected from the lobes of 3C 353 was non-thermal in origin, being consistent with the IC/CMB model prediction for the lobes if the magnetic field strength is slightly below the equipartition value, $U_{\mathrm{B}} \lesssim U_{\mathrm{e}}$.

The present paper is organized as follows. In $\S 2$, we describe the Chandra observations we performed and the data reduction process, and give a brief overview of the X-ray image we have obtained. A detailed analysis of the jet structures based on the X-ray/radio maps is presented in $\S 3$, and spectral analyses of the nucleus, jets, and lobes are given in $\S 4$. In $\S 5$, we discuss our findings in the context of various jet emission/particle acceleration models. The final conclusions are presented in $\S 6$. Throughout this paper we adopt a modern cosmology with $\Omega_{\mathrm{m}}=0.27, \Omega_{\Lambda}=0.73$ and $H_{0}=71 \mathrm{~km} \mathrm{~s}^{-1} \mathrm{Mpc}^{-1}$, leading to a luminosity distance of $d_{\mathrm{L}}=131.6 \mathrm{Mpc}$ and a conversion scale of $0.60 \mathrm{kpc} /{ }^{\prime \prime}$ for the 3C 353 redshift, $z=0.0304$.

\section{OBSERVATIONS AND DATA REDUCTION}

Before going into the details of the Chandra X-ray data, let us first remind the reader of the appearance of the jet of $3 \mathrm{C} 353$ in high resolution radio maps. Figure 1 shows NRAO Very Large Array (VLA) images of 3C 353 produced by Swain (1996). The upper panel is a deep-cleaned L-band $(1.4 \mathrm{GHz})$ image with a resolution (FWHM of restoring convolving Gaussian) of $1.3^{\prime \prime}$, similar to that of the Chandra Xray image. The lower panel shows a zoom in on the eastern jet region taken at $8.4 \mathrm{GHz}$ (X-band; $0.44^{\prime \prime}$ resolution). Full details of the data reduction are described by Swain (1996). Note that the jet is well resolved and clearly extended in the transverse direction, as compared to the unresolved nucleus. Our goal in this section is to find out how the Chandra Xray image differs from, or agrees with, these radio images and provide one-to-one identification of various jet structures. Although $3 \mathrm{C} 353$ is one of the brightest $3 \mathrm{C}$ sources, on the parsec-scale it is not as noteworthy. A "snap-shot" Very Long Baseline Array (VLBA) observation at $4.8 \mathrm{GHz}$ only detected the source on the shortest baselines. Fomalont et al. (2000) conclude the core of $3 \mathrm{C} 353$ is only $\sim 150 \mathrm{mJy}$, with a size of $\sim 15$ milli-arcseconds.

3C 353 was observed with Chandra in July 2007 with a total (requested) duration of $90 \mathrm{ks}$ (SeqNum 701549; ObsID

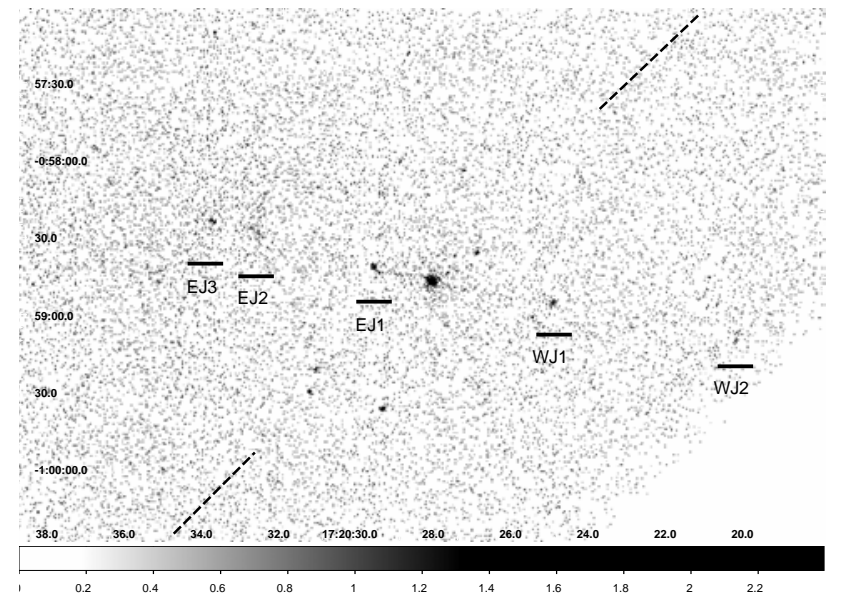

FIG. 2.- A raw X-ray image of 3C 353 obtained with Chandra for in the $0.4-8.0 \mathrm{keV}$ energy band. Five jet regions detected with WAVDETECT on angular scales of $0.5^{\prime \prime}-8^{\prime \prime}$ (wavelet radii of $1,2,4,8$, and 16 pixels) are labelled as EJ1, EJ2, EJ3, WJ1, and WJ2, respectively (see also Table1). Dashed lines show the direction of readout streak of the ACIS-S3 CCD chip, showing that the bright nucleus does not affect measurements of the jet features.

$7886,8565)$. The source was observed using the standard ACIS-S configuration, where the back-illuminated S3 chip was used to obtain maximal sensitivity to soft photons. After deleting a high background interval at the end of ObsID 7886 (Jul 04 18:22:16-18:30:36 UT), the exposure times for the two observations were $70.4 \mathrm{ks}$ (ObsID 7886) and $17.9 \mathrm{ks}$ (ObsID 8565), respectively. The active nucleus was at the aim point, resulting in both sub-arcsecond resolution and good spectral sensitivity for the whole jet (including knots, hotspots and lobes). The roll angle was constrained to preclude the possibility that any readout streak from the nucleus

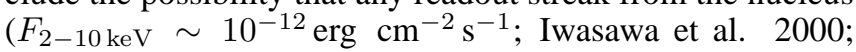
G08; see also \$4.1) could affect the imaging of the jet. Although the imaging capability of the High Resolution Mirror Assembly (HRMA) degrades substantially ( $\left.\geq 1^{\prime \prime}\right)$ for sources more than $3^{\prime}$ off-axis ${ }^{9}$, the jet in $3 \mathrm{C} 353$ extends over about $\pm 2^{\prime}$ in length, so that the $50 \%$ encircled energy for a point source is contained within a diameter of $\sim 1^{\prime \prime}$ all along the $3 \mathrm{C} 353$ outflows $^{10}$. The level-2 data were reprocessed using the CXCDS software CIAO 3.4 and CALDB 3.4.3. We generated a clean data set by selecting the standard $A S C A$ grades

\footnotetext{
9 See "Proposers' Observatory Guide v.10" at http: //asc.harvard.edu/proposer/POG/.

${ }^{10} \mathrm{See}$, in this context, $\S 3.2$, for a detailed simulation using MARX soft-
} 
$(0,2,3,4$ and 6$)$ and energy band $0.4-8.0 \mathrm{keV}$.

A co-added, raw image of the 3C $353 \mathrm{X}$-ray jet in the $0.4-8.0 \mathrm{keV}$ bandpass is shown in Figure 2. The dashed lines show the direction of the readout streak of the ACIS-S3 CCD chip, confirming that the bright nucleus does not affect our observations of the jet. The Chandra image shows that the Xray emission consists of a bright nucleus, jet-knots, hotspots and diffuse emission possibly associated with the East radio lobe. Using a wavelet decomposition source-detection algorithm (WAVDETECT; Vikhlinin et al. 1995) to detect emission enhancements on angular scales of $0.5^{\prime \prime}-8^{\prime \prime}$ (wavelet radii of $1,2,4,8$, and 16 pixels) with more than $3 \sigma$ significance, we find a total of 31 distinct X-ray sources or enhancements with a signal threshold $10^{-6}$ (the threshold for identifying a pixel as belonging to a source ${ }^{11}$ ) in the analysis region on the ACIS-S3 chip ${ }^{12}$. Among these, the brightest one is the nucleus of 3C 353, but five sources are closely aligned with radio-knots in 3C 353 as marked in Figure 2 (denoted as EJ1, $\mathrm{EJ} 2, \mathrm{EJ} 3, \mathrm{WJ} 1, \mathrm{WJ} 2)$. The statistical significance of these features are $13.7,3.8,8.2,10.0$, and $4.8 \sigma$, respectively (see Table 1). Most strikingly, the X-ray image clearly shows not only the East (main) jet-knots, but also a bright knot in the West counterjet (WJ1), that seems to be identified with the CJ2 radio knot as given in Swain et al. (1998). Also X-ray emission near the West hotspot region is detected in the image (WJ2).

Since there are some X-ray point sources in the field, it is important to ask whether the the counterjet feature (WJ1) detected in X-rays could just be a chance coincidence with a background or foreground object. Of the 26 sources without radio counterparts in the analysis region, 13 were coincident with optical stars in our Galaxy listed in the USNOB1.0 catalogue (Monetet al. 2003) ${ }^{13}$, and 13 were unidentified or newly found in this Chandra X-ray observation. We also checked the NASA/IPAC Extragalactic Database ${ }^{14}$ and found four galaxies within a $5^{\prime}$ radius of $3 \mathrm{C} 353$, but none of these was detected in our Chandra X-ray image. This leads us to conservatively estimate a probability that the $\mathrm{X}$-ray source detected in the vicinity of $\mathrm{CJ} 2$ (within $3^{\prime \prime}$ radius) is just a chance coincidence (i.e., not related to the jet) is $\leq 0.4 \%$. An even stronger argument for WJ1 being different from the background point sources is that it is well resolved and broader than the point spread function, as we will show in $\S 3.2$ (see also Figure 9).

Figure 3 shows an exposure corrected image of 3C 353 in the energy band $0.4-8.0 \mathrm{keV}$, with $1.4 \mathrm{GHz}$ radio contours $\left(1.2,4.6,8.1\right.$, and $\left.11.5 \mathrm{mJy}_{\text {beam }}{ }^{-1}\right)$ overlaid. The X-ray image has been smoothed with a two-dimensional Gaussian function with $\sigma=1.5$ pixels (1 Chandra pixel is $0.492^{\prime \prime}$ ) using DS9 version 4.0. The nucleus is located in the center of the image, and the jet extends to the East (main jet) and the West (counter jet) directions. Figure 4 shows the central region of $3 \mathrm{C} 353$. The East jet (EJ1 and EJ2) shows rather complicated features with multiple enhancements or peaks even inside each source region. We therefore re-examined the source-detection algorithm by running WAVDETECT with a single wavelet radius, changing its value from 1 to 16 pix-

\footnotetext{
11 See http: / / cxc.harvard.edu/ciao3.4/download/doc/detect

12 A square region with physical (detector) coordinates $3500 \leq \mathrm{DETX} \leq 4500$ and $3600 \leq \mathrm{DETY} \leq 4600$, where the nucleus of $3 \mathrm{C} 353$ is at $(4086.7,4131.6)$.

13 See also http://www. nofs.navy.mil/data/fchpix/for a Web version of the catalogue.

14 NED; see http://nedwww.ipac.caltech.edu/.
}

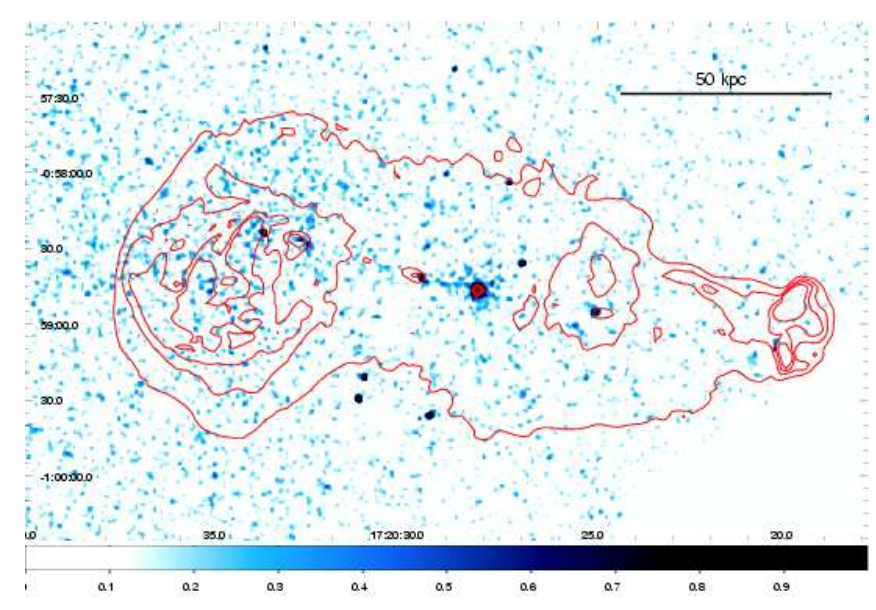

FIG. 3.- An exposure corrected X-ray image of 3C 353 (Chandra ACISS3: $0.4-8.0 \mathrm{keV})$ smoothed with a two-dimensional Gaussian function with $\sigma=1.5$ pixels using DS9, overlaid with radio contours (VLA, $1.4 \mathrm{GHz}$ ). The contour levels are $1.2,4.6,8.1$, and 11.5 mJy beam $^{-1}$

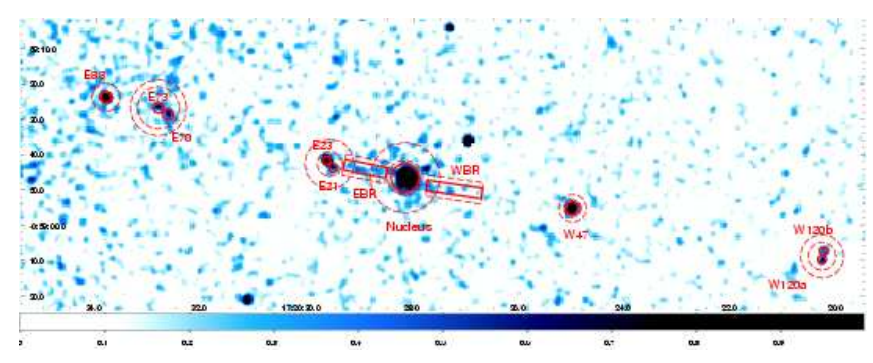

FIG. 4.- A Chandra image of the central region of 3C 353, smoothed with a two-dimensional Gaussian function with $\sigma=1.5$ pixels using DS9 $(0.4-8.0 \mathrm{keV})$. The areas indicated by solid lines mark regions from which $\mathrm{X}$-ray spectra were extracted and modeled with a power-law function plus Galactic absorption (Table 1). Backgrounds were measured from the surrounding dashed annuli or boxes.

els. Interestingly, these jet features were repeatedly detected at various wavelet radii, suggesting that there is relatively narrowly peaked (i.e., point-source-like) emission embedded in an extended component, and/or there is another peak of emission very close to the primary peak. Furthermore, we note that the centroid of each detected regions exhibits a gradual shift as we move to larger wavelet radii, although the positions are in good agreement for background point sources. Unfortunately, the photon statistics are not good enough to fully describe the complexity of the detected jet features. We therefore approximate the $\mathrm{EJ} 1$ and $\mathrm{EJ} 2$ regions below as a composite of two emission peaks, as indicated in Figure 4 (denoted as E21/E23 and E70/73, respectively). Similarly, the EJ3 and WJ1 regions are denoted as E88, W47 according to the distance from the nucleus of 3C 353 (see Table 1 and Figure 4). A weak but rather complicated X-ray feature (W120 a,b: Figure 4) is also detected near the West (counterjet) termination region, but its nature is not obvious because it is rather close to the edge of the S3 CCD chip in the ACIS-S array. Net photon counts from these jet-related structures, after subtracting the background photons, are given in Table 1 . In addition to the bripgliantúcleus and knot-like (jet or hotspot-related) features, we can see weak diffuse emission that bridges the innermost jet knots and the nucleus, and that is only visible in the X-ray image (regions marked as EBR and WBR in Figure 4, detected at the $\sim 3 \sigma$ level). No counterpart to this structure was found in the $1.4 \mathrm{GHz}$ radio map. 


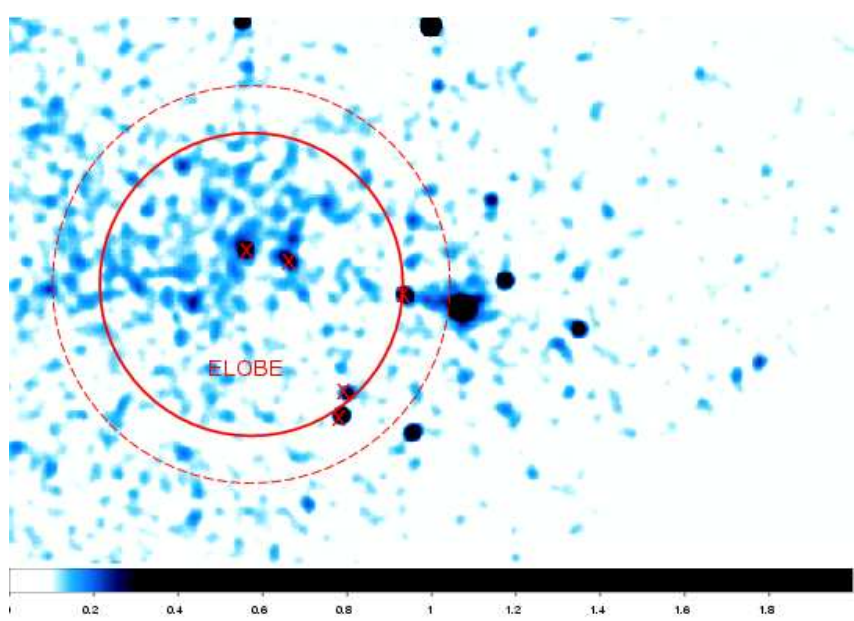

FIG. 5.- A Chandra image of the lobe region of 3C 353, smoothed with a two-dimensional Gaussian function with $\sigma=4$ pixels using DS9 (0.4-8.0 $\mathrm{keV})$. The areas indicated by solid lines mark regions from which spectra of lobe X-ray emission were extracted and modeled with a power-law function plus Galactic absorption (Table 4). The backgrounds was measured from the dashed annulus. Crosses show the positions of point sources (jet knots and background point sources) which were excluded when extracting the lobe spectrum.

Figure 5 shows a more heavily smoothed image (smoothed with a Gaussian with $\sigma=4$ pixels) of 3C 353. Some excess emission was found at the East lobe region, which corresponds to the detection of this region by G08, but no emission was detected exceeding the background level at the location of the West radio lobe in our Chandra image. This is simply due to the relatively poor photon statistics of Chandra compared to XMM-Newton; the expected flux in the West lobe region is less than a quarter of that of the east lobe region and hence is difficult to detect with our Chandra exposure. Although the primary purpose of this paper is to present the properties of the X-ray emission associated with the 3C 353 jet, we will also briefly present the analysis of the bright nucleus and East radio-lobe in later sections of the paper in order to compare our results with those obtained recently with XMM-Newton (G08).

\section{IMAGE ANALYSIS}

In order to understand the origin of X-ray emission associated with the 3C 353 jet knots, we first derive the intensity (photon counts) profile of the jet both in the longitudinal and transverse directions, which enables the direct comparison of $\mathrm{X}$-rays from 3C 353 with the high resolution VLA images (1.4 GHz and 8.4 GHz: Swain et al. 1998). This allows us to study in detail the positional offsets between the radio and $\mathrm{X}$-ray peaks, and differences in the jet width, as measured perpendicular to the main jet axis. Details of the radio/X-ray morphology for different jet features are shown again in Figure 6 (see also Table 1 for the nomenclature of radio and X-ray jet knots).

\subsection{Longitudinal Jet Profile: Positional Offset}

We first integrated the photon counts in a rectangular region of $\pm 12.8^{\prime \prime}$ width around the main jet axis. Then the longitudinal intensity profiles were constructed from $x=-150^{\prime \prime}$ to $+150^{\prime \prime}$ (defined from the east to the west, where $x=0$ represents the position of the nucleus). Figure 7 shows the longitudinal jet profiles thus produced in both radio and X-ray. This profile confirms the detection of jet knots (E21, E23, E70,
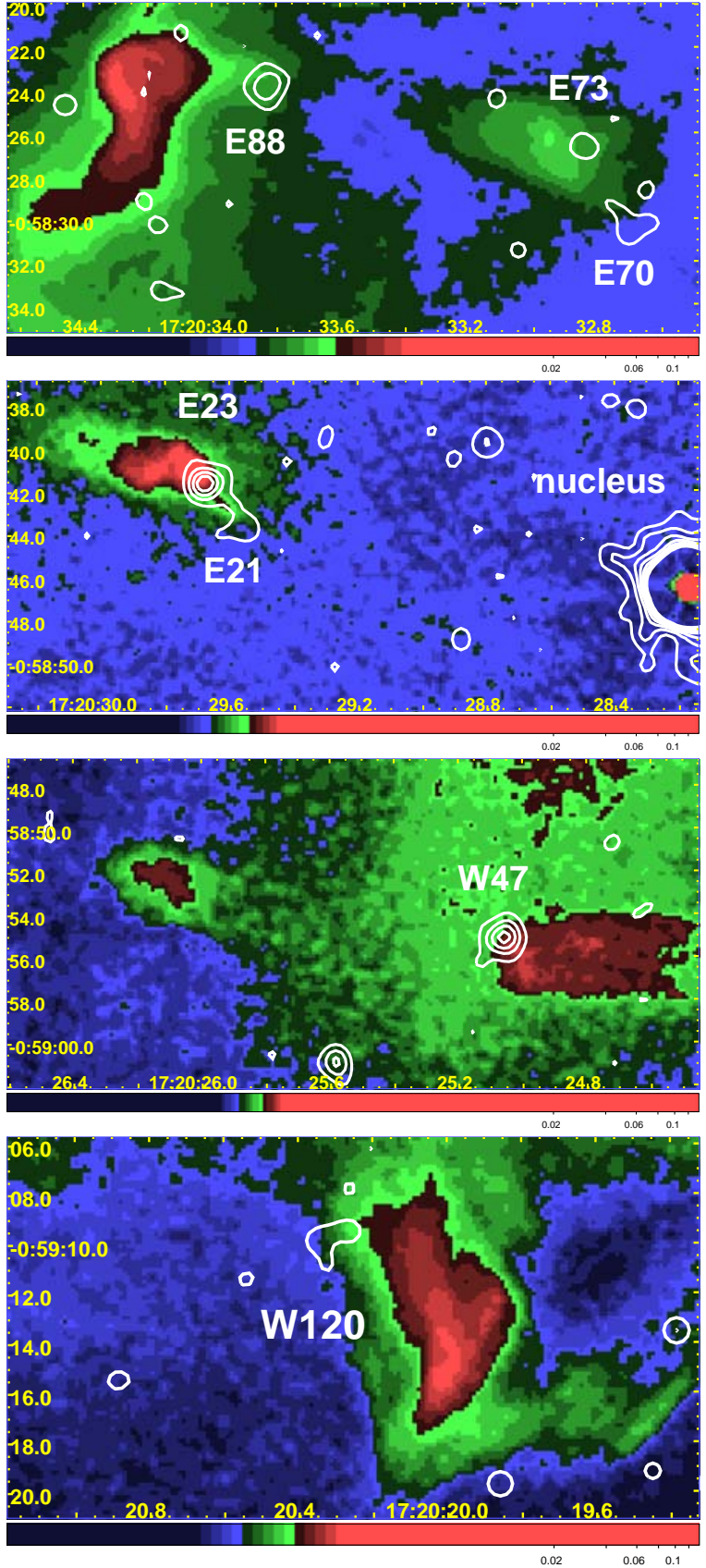

FIG. 6.- Close-ups of the 3C 353 radio jets at $8.4 \mathrm{GHz}$ with 0.4 " beam FWHM (colors), overlaid with the X-ray contours produced from a smoothed (FWHM=1") flux map between 0.8 and $7 \mathrm{keV}$. The contours start at $1.5 \times 10^{-17} \mathrm{erg} \mathrm{cm}^{-2} \mathrm{~s}^{-1}$ per 0.246 " pixel, and increase by $1.5 \times 10^{-17} \mathrm{erg} \mathrm{cm}^{-2} \mathrm{~s}^{-1}$ up to $12 \times 10^{-17} \mathrm{erg} \mathrm{cm}^{-2} \mathrm{~s}^{-1}$.

E73, W47), of X-ray features preceding the radio terminal regions (E88, W120a,b), and finally of excess emission in the East lobe regions. In the radio profiles, the intensity gradually increases toward the terminal hotspots, but the same trend is not so clearly observed in the X-ray intensity profile.

Figure 7 also makes it clear that there are substantial offsets between X-ray and radio knots/hotspots, with the centroids of the X-ray peaks being closer to the nucleus, a phenomenon that has previously been observed in several other jet sources studied with Chandra (see, e.g., Hardcastle et al. 2003; Siemiginowska et al. 2002; but see also Siemiginowska et al. 2007 for the revised analysis using new high S/N Chan- 


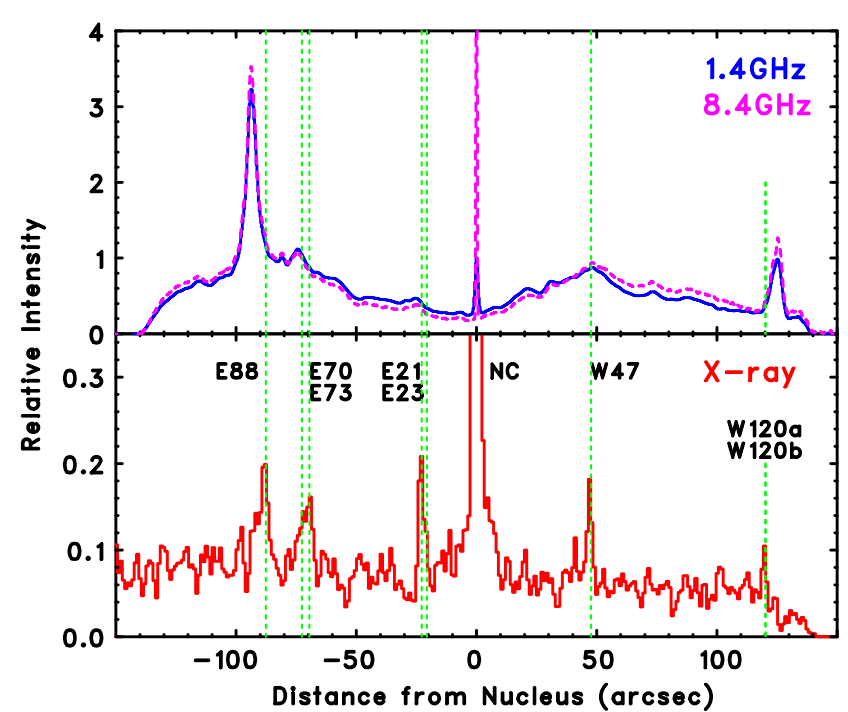

FIG. 7.- Intensity profiles along the major axis of the lobes of 3C 353 . Above: the radio emission at $1.4 \mathrm{GHz}$ (blue) and $8.4 \mathrm{GHz}$ (magenta), Below: the $\mathrm{X}$-rays in the $0.4-8.0 \mathrm{keV}$ range. Intensities of radio/X-rays emissions are normalized to their nuclear fluxes $(\times 1$ for $1.4 \mathrm{GHz}, \times 10$ for $8.4 \mathrm{GHz}$ and $\mathrm{X}$-rays). The green dotted lines show the centers of jet knots and hotspots, which are defined in Figure 4.

dra data). Figure 8 shows the positional offset between radio and $\mathrm{X}$-ray peaks of the various jet knots and hotspot as a function of the distance from the nucleus. To measure the offsets we have used the $1.4 \mathrm{GHz}$ map, since its resolution is $1.3^{\prime \prime}$, close to the $\sim 1^{\prime \prime}$ resolution of the Chandra X-ray image. Clearly, the measurements imply a smooth increase of offsets downstream along the jet, which possibly saturates at a maximum value at the East hotspot (E88; $6.7 \pm 0.2^{\prime \prime}$, consistent with the value measured using XMM-Newton by G08). ${ }^{15}$ The maximum observed offset $\left(\sim 7^{\prime \prime}\right)$ corresponds to large physical distance $(\sim 4 \mathrm{kpc})$ at the distance of $3 \mathrm{C} 353$. It is important to mention in this context that there is no apparent mismatch or discrepancy of this trend between the main jet (E23, E70, E88) and counterjet (W47) suggesting the same physical origin for the X-ray production (see $\$ 5.2$ for detailed discussion). Although the X-ray emission from the hotspot region is coincident with the start of the increase in the radio, the good (i.e., statistically significant) detection of the X-rays is more than $\sim 7^{\prime \prime}$ upstream from the peak of the radio emission from the East hotspot. There is some weak radio emission closer to E88, so that it is plausible that E88 may not be a direct counterpart of the East hotspot, but rather a jet knot like the E23 and E73 X-ray features. This idea would be supported by the relatively similar spectral energy distributions (SEDs) of various jet knots, as we will see in $\S 4.2$. Similarly, the Xray emission detected near the West hotspot (W120a,b) may not be related directly to the jet-termination structure in the radio. These open issues cannot be addressed even using the excellent resolution of both the Chandra and VLA images.

\subsection{Transverse Jet Profile: Jet Width}

We next integrate the photon counts across the jet at certain distances from the nucleus to construct transverse intensity profiles of the jets. Here we concentrate on the profiles of the relatively bright X-ray jet knots, E23, E73, and W47, perpendicular to the main jet axis. We define the regions of

15 The X-ray feature W120 is not considered to quantitatively estimate an offset, since it lacks any obvious radio counterpart.

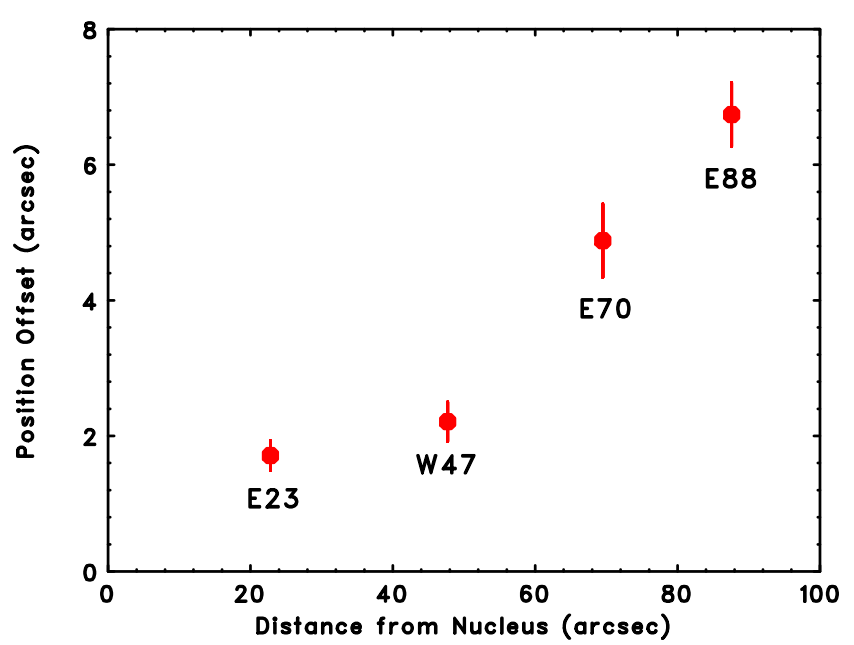

FIG. 8.- Positional offset of jet knots and hotspots between radio and

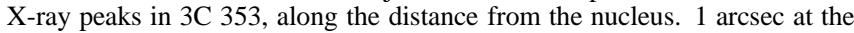
distance of 3C 353 corresponds to $0.60 \mathrm{kpc}$. In this figure the X-ray feature W120 was not included, since it lacks any obvious radio counterpart.

integration to be small boxes ( $3^{\prime \prime}$ along the main jet axis) centered on each jet knot, so as to reduce the contamination from neighboring fainter jet knots, E21 and E70. Figure 9 shows the transverse jet profiles thus produced for (a) E23, (b) E73, and (c) W47 as compared with the corresponding radio intensity profiles taken from the same regions. In each panel, sub-panels show the profiles after subtracting smooth variations of underlying lobe emission. The peak of the intensity profile is normalized to unity in each panel for convenience of comparison of the radio and X-ray profiles.

As discussed in Swain (1996) and Swain et al. (1998), the width of the radio jet in all these figures is much broader than the resolution of the radio map $\left(1.3^{\prime \prime}\right.$ for $1.4 \mathrm{GHz}$ and $0.44^{\prime \prime}$ for $8.4 \mathrm{GHz}$ ). The transverse profile in the radio cannot be well represented by a simple Gaussian function, but instead shows a flat-topped profile which is especially clear in E73 and W47. For quantitative comparison, the width of the jet, at which the intensity becomes half of the maximum (FWHM), are 3.16" $/ 2.66^{\prime \prime}$ (E23), 4.00" $/ 3.77^{\prime \prime}$ (E73), and $3.43^{\prime \prime} / 3.32^{\prime \prime}$ (W47), where the widths are quoted for the $1.4 \mathrm{GHz}$ and $8.4 \mathrm{GHz}$ radio maps respectively (see Table 2). This flat-topped profile is characteristic of a situation in which the bulk of the jet radio emission is produced within the jet boundary (shear) layer, especially in the case of the counterjet knot W47 (corresponding to the radio feature CJ2 in Swain et al. 1998).

In contrast, the X-ray knots show no evidence for the flattopped profiles seen in the radio images and are instead well represented by a smooth Gaussian function. To investigate any possible broadening of the point spread function (PSF) at the position of each jet knot, we performed Monte-Carlo simulations ${ }^{16}$ using MARX 4.0. We modeled a point source with a power-law X-ray spectrum with a photon index $\Gamma=1.7$, modified by Galactic absorption $N_{\mathrm{H}}=1.6 \times 10^{21} \mathrm{~cm}^{-2}$, as indicated from modeling the observed X-ray spectra of the nucleus and jet knots (see $\$ 4.2$ ). The resultant PSF is shown as a dotted green line in Figure 9. The FWHM of the X-ray jet (E23, E73) and the counterjet (W47) are broader than that predicted by the PSF and hence the jet is well resolved in X-rays. In fact, the observed FWHM of the X-

\footnotetext{
${ }^{16}$ See http: //space/mit.edu/ASC/MARX/marx_4 .0_manual.pdf.
} 

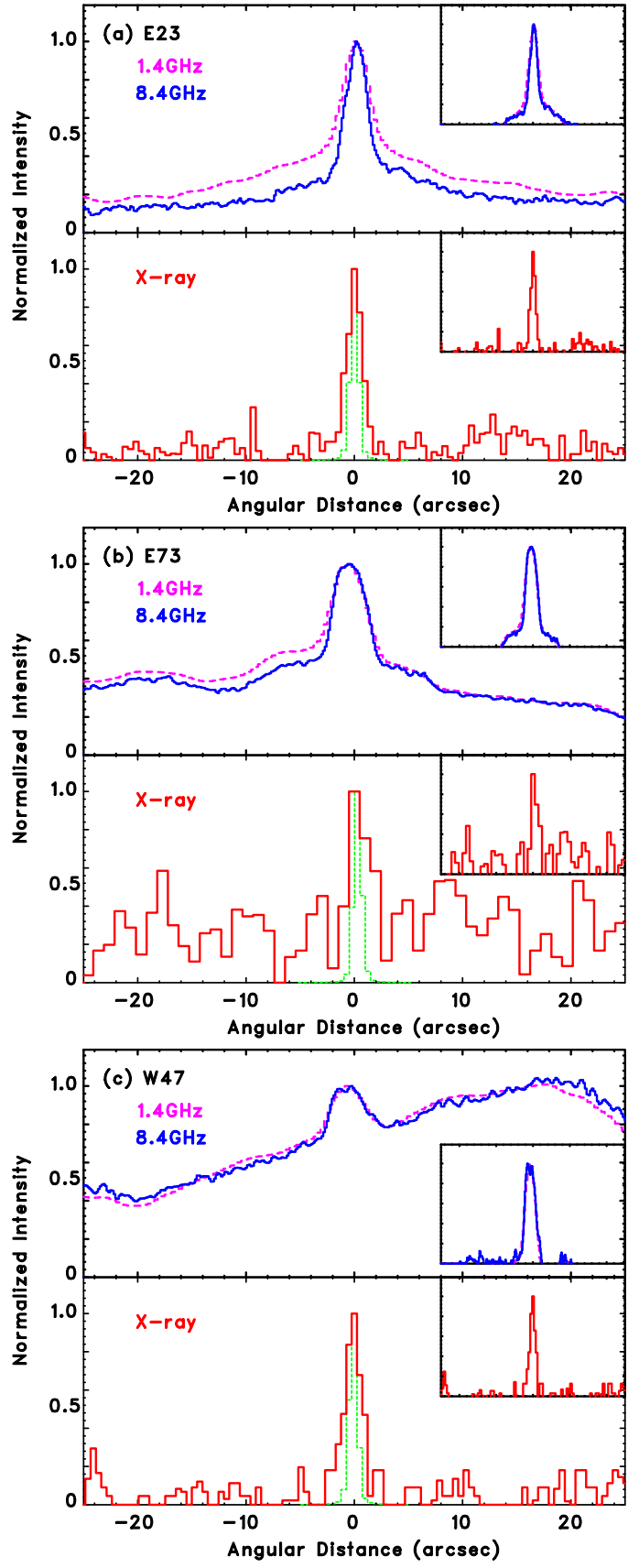

FIG. 9.- (a) Comparison of transverse profile in the jet knot E23. Above: the radio emission at $1.4 \mathrm{GHz}$ (blue) and $8.4 \mathrm{GHz}$ (magenta). Below: the $\mathrm{X}$-rays in the $0.4-8.0 \mathrm{keV}$ range. A green dotted line shows the point spread function at the off-axis distance of the source simulated with MARX, by assuming a source spectrum with photon index $\Gamma=1.7$ modified by Galactic absorption $N_{\mathrm{H}}^{G}=1.6 \times 10^{21} \mathrm{~cm}^{-2}$. In each panel, the insets to the upper right present the jet profiles after subtracting the smooth variation of the underlying lobe emission. Radio resolutions are the same as in Figure 1. (b) Same as (a), but for the jet knot E73. (c) Same as (a), but for the jet knots W47.

ray jets are $1.65^{\prime \prime} \pm 0.27^{\prime \prime}$ (E23), $1.98^{\prime \prime} \pm 0.48^{\prime \prime}$ (E73), and $1.79^{\prime \prime} \pm 0.36^{\prime \prime}$ (W47), respectively, as compared to the PSF width of $0.93^{\prime \prime} \pm 0.01^{\prime \prime}$ (Table 2). Although the difference between the radio and X-ray jet widths is still marginal ( $\mathrm{a} \gtrsim 4 \sigma$ effect even for the most significant case, the jet knot W47), these results provide important information about the internal jet structures and the underlying jet physics, as we will discuss in $\S 5.3$.
TABLE 2

WIDTH OF 3C 353 JET KNOTS AS MEASURED FROM RADIO/X-RAY IMAGES.

\begin{tabular}{lcccc}
\hline Name & $\begin{array}{c}\text { beams width }^{a} \\
1.4 \mathrm{GHz} / 8.4 \mathrm{GHz} / \mathrm{X} \text {-ray } \\
\end{array}$ & $\Delta w_{1.4}^{b}$ & $\Delta w_{8.4}^{b}$ & $\Delta w_{\mathrm{X}}^{c}$ \\
& {$\left[{ }^{\prime \prime}\right]$} & {$\left[^{\prime \prime}\right]$} & {$\left[^{\prime \prime}\right]$} & {$\left[^{\prime \prime}\right]$} \\
\hline E23 & $1.3 / 0.44 / 0.92$ & 3.16 & 2.66 & $1.65 \pm 0.27$ \\
E73 & $1.3 / 0.44 / 0.94$ & 4.00 & 3.77 & $1.98 \pm 0.48$ \\
W47 & $1.3 / 0.44 / 0.93$ & 3.43 & 3.32 & $1.79 \pm 0.36$ \\
\hline
\end{tabular}

Note.: all the errors are $1 \sigma$.

aFWHM width of the circular Gaussian beams which was used to restore the $1.4 \mathrm{GHz} / 8.4 \mathrm{GHz}$ radio images; the X-ray width is estimated from the Gaussian dispersion obtained from a simulation using MARX at various offset angles (see $\S 3.2$ for detailed description of the X-ray simulation).

${ }^{b}$ FWHM $1.4 \mathrm{GHz} / 8.4 \mathrm{GHz}$ width of various jet knots measured by using the projected profiles perpendicular to the main jet axis from the deconvolved radio images presented in Figure 1.

${ }^{c}$ FWHM width of various jet knots measured from the $0.4-8.0 \mathrm{keV}$ Chandra image.

\section{SPECTRAL ANALYSIS}

We next present detailed results from the spectral analysis of 3C 353's nucleus, jet knots and radio lobe. We find that the nucleus shows a complex X-ray spectrum with a heavily absorbed power-law component, while both the jets and radio lobe are well represented by a simple power law moderated by Galactic absorption. We find a smooth variation of radio $(1.4 \mathrm{GHz})$ to $\mathrm{X}$-ray $(1 \mathrm{keV})$ flux density ratio as a function of distance from the nucleus for the jet. Finally, the spectral energy distributions of several bright jet knots are presented in order to investigate the origin of the X-ray emission from the counterjet knot W47.

\subsection{Nucleus}

We extracted the spectrum of the nucleus from a circular region with a radius of $4^{\prime \prime}$, while the background was estimated from a local background region with an annulus of an outer radius of $10^{\prime \prime}$ and inner radius of $5^{\prime \prime}$ (see also Figure 4). We obtained $2833 \pm 54$ net photons from the nucleus, combining data from ObsIDs 7886 and 8565. During our Chandra observations, no variability was detected for the nucleus on day-toweek scale with a constant $\mathrm{X}$-ray count rate of $\simeq 0.03 \mathrm{cts} \mathrm{s}^{-1}$ in the $0.4-8.0 \mathrm{keV}$ energy band. For the spectral fitting, an accurate estimate of the Galactic absorption $\left(N_{\mathrm{H}}^{G}\right)$ is obviously important. At this point, we note that the column density towards 3C 353 is uncertain, as has already been pointed out in the literature. Iwasawa et al. (2000) adopted a value of $1.0 \times 10^{21} \mathrm{~cm}^{-2}$ based on the HI measurements of Dickey \& Lockman (1990). The Dickey \& Lockman value was questioned by G08, based on the Galactic dust measurements of Schlegel et al. (1998), which would provide a column density of a factor of $\sim 2$ higher $\left(\sim 2.6 \times 10^{21} \mathrm{~cm}^{-2}\right)$. G08 argued that the true value is likely to lie somewhere between these two extremes; they used their new XMM-Newton data to directly estimate a column density from background sources and found a weighted mean $N_{\mathrm{H}}^{G}=(1.64 \pm 0.07) \times 10^{21} \mathrm{~cm}^{-2}$. In this paper, we follow this 'latest' estimate of the $N_{\mathrm{H}}^{G}$ value, fixed at $1.6 \times 10^{21} \mathrm{~cm}^{-2}$, in our spectral analysis.

We first attempted to fit the nuclear spectrum with a single power-law function modified by Galactic absorption only. However, the fits were very poor: $\chi^{2}=650$ for 221 degrees of freedom (see Table 3 ). An attempt to fit with a ther- 
TABLE 3

RESUlts OF THE Chandra SPECTRAL FITS TO THE NUCLEUS IN 3C 353.

\begin{tabular}{|c|c|c|c|c|c|c|c|c|}
\hline Model & $\overline{\Gamma_{1}}{ }^{a}$ & $\begin{array}{l}F_{1 \mathrm{keV}}{ }^{b} \\
{[\mathrm{nJy}]}\end{array}$ & $\begin{array}{l}F_{0.5-5 \mathrm{keV}^{c}} \\
{\left[10^{-15} \mathrm{cgs}\right]}\end{array}$ & $\begin{array}{l}N_{\mathrm{H}}{ }^{d} \\
{\left[10^{22} \mathrm{~cm}^{-2}\right]}\end{array}$ & $\Gamma_{2}{ }^{e}$ & $\begin{array}{l}F_{1 \mathrm{keV}}{ }^{b} \\
{[\mathrm{nJy}]}\end{array}$ & $\begin{array}{l}F_{0.5-5 \mathrm{keV}^{c}} \\
{\left[10^{-15} \mathrm{cgs}\right]}\end{array}$ & $\chi^{2}$ (dof) \\
\hline$\overline{\text { PL1 }}$ & $-0.76 \pm 0.03$ & $2.74 \pm 0.13$ & $202 \pm 4$ & $\cdots$ & .. & 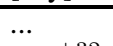 & $\cdots$ & $2.94(221)$ \\
\hline PL2 & $\ldots$ & $\ldots$ & $\ldots$ & $5.34_{-0.38}^{+0.40}$ & $1.47 \pm 0$ & $128_{-25}^{+32}$ & $964_{-11}^{+13}$ & $1.19(220)$ \\
\hline PL1+2 & $1.62 \pm 0.13$ & $14.3_{-4.4}^{+4.8}$ & $11.2 \pm 1.9$ & $6.25_{-0.39}^{+0.40}$ & $1.62 \pm 0.13$ & $195_{-42}^{+55}$ & $1140_{-140}^{+170}$ & $1.02(219)$ \\
\hline
\end{tabular}

Note. - Note: all the errors are $1 \sigma$. Galactic absorption is fixed at $N_{\mathrm{H}}^{G}=1.6 \times 10^{21} \mathrm{~cm}^{2}$ (see G08).

${ }^{a}$ : Photon index of power-law component 1 (modified by Galactic $N_{\mathrm{H}}^{G}$ only).

${ }^{b}$ : Absorption corrected flux density measured at $1 \mathrm{keV}$, in units of nJy.

c: Absorption corrected flux in $0.5-5 \mathrm{keV}$, in units of $10^{-15} \mathrm{erg} \mathrm{cm}^{-2} \mathrm{~s}^{-1}$.

${ }^{d}$ : Intrinsic absorption $N_{\mathrm{H}}$ at the distance of $3 \mathrm{C} 353(z=0.0304)$, in units of $10^{22} \mathrm{~cm}^{-2}$.

${ }^{e}$ : Photon index of power-law component-2 (modified by intrinsic $N_{\mathrm{H}}$ ).

mal bremsstrahlung model gave an even worse result, with $\chi^{2}=2254$ for 221 degrees of freedom (dof). We therefore fitted a model by adding an intrinsic absorption column density $N_{\mathrm{H}}$ at the distance of the source $(z=0.0304)$. The fitting statistic was substantially improved, such that $\chi^{2}=261$ for 220 dof, but still unacceptable in the sense that $\chi^{2}$ probability is less than $5 \%\left(P\left(\chi^{2}\right)=3 \%\right)$. Hence we adopted a model consisting of a power-law modified by $N_{\mathrm{H}}^{G}$ only, plus an another power-law heavily absorbed by an intrinsic $N_{\mathrm{H}}$. We constrained the photon indices of both power-law components to the same value, so as to reduce the number of free parameters. This model represents the observed X-ray spectra very well, with $\chi^{2}=223$ for 219 dof $\left(P\left(\chi^{2}\right)=41 \%\right.$ ). A summary of the spectral fitting parameters are shown in Table3, and the resultant best-fitting spectra are presented in Figure 10.

The power-law indices determined by our Chandra observations are consistent with those reported in G08 using the $X M M$-Newton data. There are two minor differences in the spectral fitting: (1) G08 determined both power-law indices $\left(\Gamma_{1}\right.$ and $\Gamma_{2}$ ) independently when fitting the spectrum, and (2) they added a thermal component $(k T=1 \mathrm{keV})$ to provide a good fit, as was required for some other FR II sources in the study of Hardcastle et al. (2006). Regarding the first point, our constrained fitting is justified because G08 obtained nearly equal best-fitting parameters for both power-law indices, $\Gamma_{1}=1.49 \pm 0.10$ and $\Gamma_{2}=1.33 \pm 0.30$, which are very close to our best-fitting value of $1.62 \pm 0.13$ (Table 3). Furthermore, our best-fitting value for the intrinsic column density, $N_{\mathrm{H}}=(6.3 \pm 0.4) \times 10^{22} \mathrm{~cm}^{-2}$, is perfectly consistent with that determined using XMM-Newton $\left(N_{\mathrm{H}}=(6.1 \pm 0.6) \times 10^{22} \mathrm{~cm}^{-2}\right)$. Although thermal emission is included in the G08 fit to the XMM-Newton data, it contributes less than $\sim 1 \%$ of flux in the energy range between 0.6 and $8 \mathrm{keV}$ (see Figure 4 of G08) and in any case may well have originated on scales larger than our Chandra extraction region given the much larger XMM-Newton PSF. We therefore do not regard the differences between the spectral models used as in any way significant.

Using our model, we determine the absorption corrected luminosity in the $2-10 \mathrm{keV}$ band for the heavily absorbed nucleus (PL2) is $(2.43 \pm 0.08) \times 10^{42} \mathrm{erg} \mathrm{s}^{-1}$. This is only $\sim 15 \%$ lower than that reported for the XMM-Newton observations during the August 2006 and February 2007 observations $\left(2.82 \times 10^{42} \mathrm{erg} \mathrm{s}^{-1}\right)$, suggesting only minor variability of the nucleus on a timescale of a year. By using the ASCA data, Iwasawa et al. (2000) reported a factor of two larger lu-

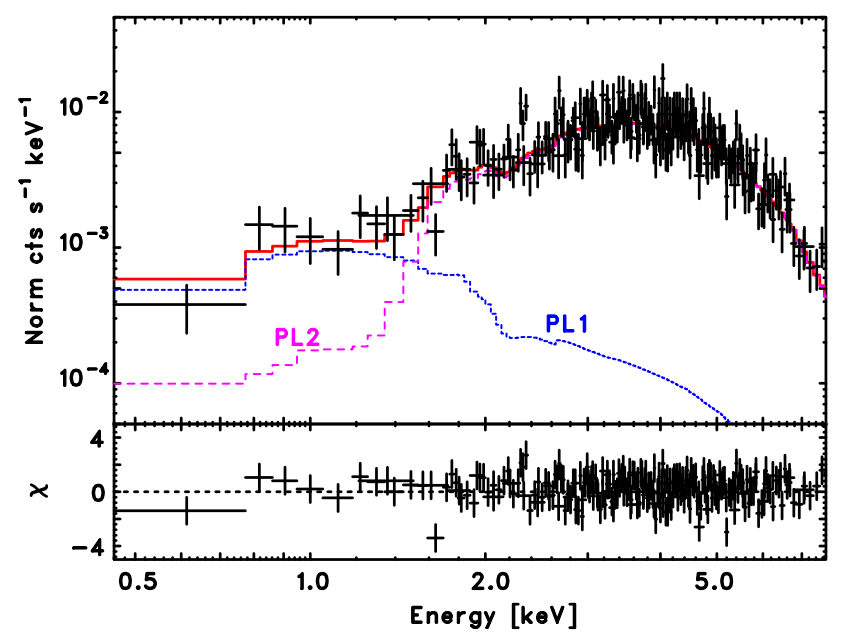

FIG. 10.- Background-subtracted ACIS-S3 spectrum of the nucleus of 3C 353. Data points are plotted as crosses; the dotted and dashed lines represent the power-law component with Galactic absorption (PL1; Table 3), and the second power-law component with redshifted intrinsic absorption (PL2), while the solid line is their sum. The bottom panel shows the residuals for the best-fit double-power law models.

minosity in the same $2-10 \mathrm{keV}$ band $\left(4.5 \times 10^{42} \mathrm{erg} \mathrm{s}^{-1}\right.$; corrected for modern cosmology with with $\Omega_{\mathrm{m}}=0.27$, $\Omega_{\Lambda}=0.73$ and $H_{0}=71 \mathrm{~km} \mathrm{~s}^{-1} \mathrm{Mpc}^{-1}$ ) in their observations taken a decade earlier, in September 1996. We suspect that contamination from the neighboring cluster $\mathrm{Zw}$ 1718.10108 may be partly the reason for this diagreement, since the PSF of ASCA $\left(\sim 3^{\prime}\right)$ is much larger than that of either XMMNewton or Chandra, but it probably also indicates some real long-term variability in the AGN X-ray output.

\subsection{Jet and Hotspots}

We next carry out a spectral analysis of various structures (including jet knots and hotspots) related to the $3 \mathrm{C} 353$ jet. The areas indicated by solid lines in Figure 4 mark regions from which spectra of the X-ray emission were extracted, while background spectra were extracted using the dashed regions. Parameters of the source extraction regions (distance from the nucleus to the center of the region, and source radius), as well as the results of spectral fitting, are summarized in Table 4. Due to the limited photon statistics, we binned the X-ray spectrum into three energy bands for both observations (ObsIDs 7886 and 8565): $0.3-1 \mathrm{keV}, 1-3 \mathrm{keV}$ and $3-10 \mathrm{keV}$. A power-law function absorbed by the Galactic column density (fixed to $N_{\mathrm{H}}=1.6 \times 10^{20} \mathrm{~cm}^{-2}$; see above) 


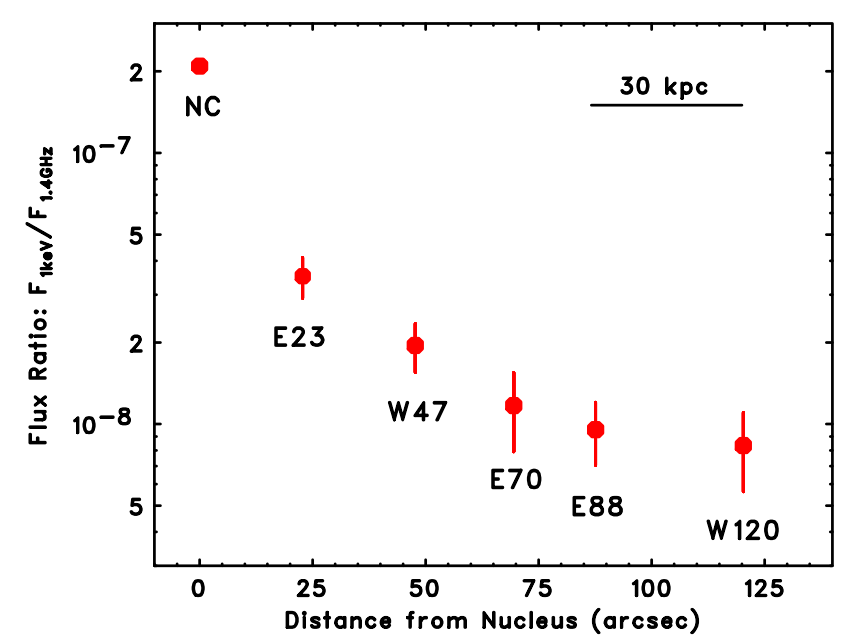

FIG. 11. - Variation of X-ray $(1 \mathrm{keV})$ to radio $(1.4 \mathrm{GHz})$ flux density ratio along the major axis of $3 \mathrm{C} 353$. Each plots involve the regions defined in Figure 4. Radio fluxes were extracted from exactly the same jet volumes as $\mathrm{X}$-ray fluxes (although the radio background regions were slightly shifted in order to avoid contamination from the bright radio structures downstream of the outflows).

gives acceptable fits with $\chi^{2}$ probability $P\left(\chi^{2}\right) \geq 15 \%$ for 4 or 5 dof. The spectral photon index was fixed at $\Gamma=1.7$ to match the radio photon index, except for the relatively bright jet knots E23/W47 and hotspot E88. (The photon index determined for these bright jet knots is consistent with 1.7 within the $90 \%$ confidence range.)

Figure 11 shows the variation of flux density ratio measured between $1 \mathrm{keV}$ and $1.4 \mathrm{GHz}, F_{1 \mathrm{keV}} / F_{1.4 \mathrm{GHz}}$, as a function of distance from the nucleus. Clearly the X-ray-to-radio flux ratio decreases downstream along the jet, as is often (though not always) observed in many other radio sources (see §5.3.2). Remarkably, this is true even for the counterjet. Although Xray counterjet detections were only made for W47 and, possibly, W120a,b, we believe this to be due to the limited sensitivity of Chandra even with our deep $90 \mathrm{ks}$ exposure. In fact, the $3 \sigma$ detection limit for this observation corresponds to a flux limit $\sim 0.1 \mathrm{nJy}$. Thus, assuming the jet-counterjet radio brightness asymmetry $\approx 2$ (Swain 1996) is also valid for the X-ray counterparts, most of the X-ray emission from the counterjet is not expected to be detected, with the exception of the brightest counterjet knot W47. We note that the flux ratio of the nucleus $\left(F_{1 \mathrm{keV}} / F_{1.4 \mathrm{GHz}}\right.$; absorption corrected) is much larger than that for the jet-related structures. This is not surprising, given that the heavily absorbed component of the nuclear X-ray emission is believed to be dominated by the accretion disk corona, while the nuclear radio flux is supposed to be produced by the unresolved, self-absorbed inner portions of the jets.

Finally, we constructed the spectral energy distributions of the bright jet knots E23, W47 and hotspot E88. For this, we took exactly same source regions for the radio and $\mathrm{X}$ ray as defined in Figure 4. We derived multi-frequency radio spectral information using radio maps obtained at four different frequencies between $330 \mathrm{MHz}$ and $8.4 \mathrm{GHz}$ (adapted from Swain et al. 1998 for $1.4 \mathrm{GHz}, 8.4 \mathrm{GHz}$ images, and G08 for $330 \mathrm{MHz}$ and $5 \mathrm{GHz}$ radio maps). Since the resolutions of the radio maps are different at these frequencies and the contamination from the lobe is non-negligible, we made several trials assuming different choices of background regions. We estimate that the uncertainty in the radio flux measurements

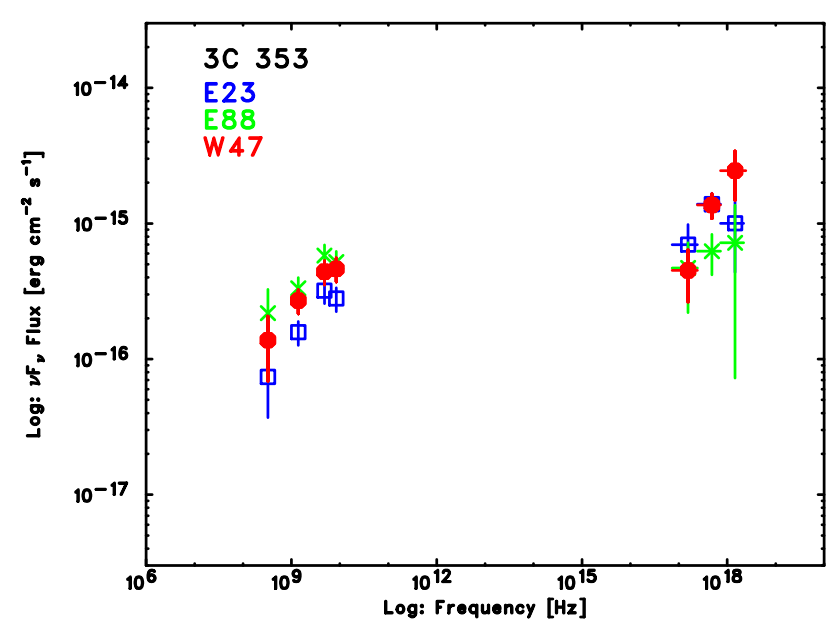

FIG. 12.- Comparison of the spectral energy distribution of the jet knot (E23 in blue and E73 in green) and the counterjet knot (W47; in red).

is typically $20-50 \%$, but this is not important for the discussion below. As before, the X-ray data were binned in three energy bands to reduce the statistical uncertainty; $0.3-1 \mathrm{keV}$, $1-3 \mathrm{keV}$, and $3-10 \mathrm{keV}$, respectively. Figure 12 compares the spectral energy distributions (SEDs) thus produced, and shows that the overall spectral features are remarkably similar to each other, suggesting that the same physical process is at work for the X-ray production in the E23, E88 and W47 knots. Except in the case of W47, the X-ray spectral index obtained with Chandra is perfectly consistent with the radio synchrotron power-law slope (radio spectral index $\alpha_{\mathrm{R}} \simeq 0.7$ ). The X-ray spectrum of W47 seems to be slightly harder, but this is only $\mathrm{a} \lesssim 2 \sigma$ effect. One may note, however, that the $\mathrm{X}$-ray spectral points cannot be connected smoothly with the extrapolation of the radio data, whether we assume either a single or a broken power-law form for the radio-to-X-ray continuum.

\subsection{Lobe}

Finally we consider the X-ray emission from the East radio lobe, for which the previous XMM-Newton observations confirm substantial diffuse emission (G08). We do not consider the West radio lobe since its emission was too weak to be detected within our Chandra exposure (see $\S 2$ ). The areas indicated by the solid line in Figure 5 mark the region from which the spectrum of the lobe X-ray emission was extracted (a circle of $r=60^{\prime \prime}$ centered on $\mathrm{RA}=17: 20: 33.694$, DEC $=-00: 58: 37.77)$, but excluding the inner jet knots $(\mathrm{E} 21 / 23$, E70/73), hotspots (E88) as well as background point sources (marked as crosses) - these were approximated as circles of $r=1.5^{\prime \prime}$ each. The dotted line shows a region of the background extraction with $r=78.7^{\prime \prime}$, excluding some background point sources. As discussed in detail by G08, the background emission from the cluster environment is the primary contributor to the uncertainty in the lobe flux density. We therefore adopted exactly the same regions for both the source and background extraction regions as those that were assumed for the $X M M$-Newton data analysis, enabling a direct comparison of the results.

A power-law model gave better fits than a thermal bremsstrahlung model, with $\chi^{2}=15.8$ for 12 dof, for the East lobe (see Table 4). The best-fitting photon index was $\Gamma=2.17_{-0.39}^{+0.43}$, which is consistent with that determined from the XMM-Newton data analysis $(\Gamma=1.9 \pm 0.4 ; \mathrm{G} 08)$. The 
TABLE 4

RESUlts OF THE Chandra SPECTRAL FITS TO THE JET KNOTS, HOTSPOTS AND LOBE IN 3 C 353.

\begin{tabular}{|c|c|c|c|c|c|c|c|}
\hline Name & $\begin{array}{l}\text { Distance }^{a} \\
\text { ["] }]^{\prime 2}\end{array}$ & $\begin{array}{l}\text { Region }^{b} \\
{\left[{ }^{\prime \prime}\right]}\end{array}$ & Net counts $^{c}$ & $\Gamma^{d}$ & $\begin{array}{l}F_{1 \mathrm{keV}}{ }^{e} \\
{[\mathrm{nJy}]}\end{array}$ & $\begin{array}{l}F_{0.5-5 \mathrm{keV}^{g}} \\
{\left[10^{-15} \mathrm{cgs}\right]}\end{array}$ & $\chi^{2}($ dof $)$ \\
\hline & & & \multicolumn{5}{|l|}{ main jet } \\
\hline EBR & 11.9 & {$[12,3]$} & $19.0 \pm 6.6$ & $1.7^{f}$ & $0.24 \pm 0.09$ & $1.56 \pm 0.61$ & $0.86(5)$ \\
\hline E21 & 20.6 & 1.2 & $10.2 \pm 3.5$ & $1.7^{f}$ & $0.09 \pm 0.04$ & $0.57 \pm 0.24$ & $0.78(5)$ \\
\hline E23 & 22.8 & 1.2 & $36.2 \pm 6.2$ & $1.81_{-0.27}^{+0.29}$ & $0.42 \pm 0.09$ & $2.60 \pm 0.47$ & $0.84(4)$ \\
\hline E70 & 69.5 & 1.5 & $11.8 \pm 4.0$ & $1.7^{f^{-0.2 t}}$ & $0.12 \pm 0.04$ & $0.80 \pm 0.29$ & $0.63(5)$ \\
\hline E73 & 72.6 & 1.5 & $9.8 \pm 3.7$ & $1.7^{f}$ & $0.08 \pm 0.04$ & $0.50 \pm 0.24$ & $0.69(5)$ \\
\hline E88 & 87.6 & 1.5 & $25.8 \pm 5.7$ & $1.79_{-0.56}^{+0.63}$ & $0.24 \pm 0.08$ & $1.46_{-0.41}^{+0.43}$ & $1.64(4)$ \\
\hline \multirow[t]{2}{*}{$\mathrm{ELOBE}^{h}$} & 83.6 & 60 & $620 \pm 71$ & $2.17_{-0.39}^{+0.43}$ & $8.82_{-1.59}^{+1.55}$ & $45.6_{-7.8}^{+8.1}$ & $1.32(12)$ \\
\hline & & & \multicolumn{5}{|l|}{ counter jet } \\
\hline WBR & 14.1 & {$[16,3]$} & $20.0 \pm 6.3$ & $1.7^{f}$ & $0.24 \pm 0.08$ & $1.59 \pm 0.54$ & $0.74(4)$ \\
\hline W47 & 47.7 & 2.0 & $37.9 \pm 6.6$ & $1.22 \pm 0.33$ & $0.31 \pm 0.09$ & $2.88_{-0.59}^{+0.55}$ & $1.08(4)$ \\
\hline $\mathrm{W} 120 \mathrm{a}^{i}$ & 120.3 & 1.2 & $10.2 \pm 3.5$ & $1.7^{f}$ & $0.09 \pm 0.04$ & $0.59 \pm 0.23$ & $0.54(5)$ \\
\hline $\mathrm{W} 120 \mathrm{~b}^{i}$ & 120.1 & 1.2 & $7.2 \pm 3.0$ & $1.7^{f}$ & $0.07 \pm 0.03$ & $0.43 \pm 0.23$ & $0.78(5)$ \\
\hline
\end{tabular}

Note. - Note: all the errors are $1 \sigma$. Galactic absorption is fixed at $N_{\mathrm{H}}^{G}=1.6 \times 10^{21} \mathrm{~cm}^{2}$ (see G08).

${ }^{a}$ :Distance of the X-ray feature from the nucleus.

${ }^{b}$ :Sizes of the source extraction regions; either the length and width of a box (EBR, WBR) or the radius of a circle (E21, E23, E70, E73, E88, ELOBE, W47, W120a, and W120b).

${ }^{c}$ : Net photon counts after subtracting the background photon counts.

${ }^{d}$ : Differential X-ray photon index.

${ }^{e}$ : Absorption corrected flux density measured at $1 \mathrm{keV}$, in units of nJy.

${ }^{f}$ : Parameters were fixed to these values rather than determined from the data.

$g$ : Absorption corrected flux in $0.5-5 \mathrm{keV}$, in units of $10^{-15} \mathrm{erg} \mathrm{cm}^{-2} \mathrm{~s}^{-1}$.

$h$ : The East lobe region defined here is exactly the same as used by G08 for the XMM-Newton analysis, except that we have masked the E70, E73, and E88 regions for our analysis.

${ }^{i}$ : Note that the detected feature is close to the CCD (ACIS-S3) edge (see Figure 2).

measured $1 \mathrm{keV}$ flux density of the east lobe is $8.82_{-1.59}^{+1.55} \mathrm{nJy}$, which is slightly smaller than, but consistent within the $1 \sigma$ error with, that determined from the XMM-Newton analysis $(11.6 \pm 1.6 \mathrm{nJy} ; \mathrm{G} 08)$. The origin of the possible (but small) discrepancy, however, is clear. In G08, only the contamination from the East hotspot (E88) was excluded to estimate the lobe X-ray emission because the remaining jet features (E21, E23, E70, E73) were not resolved from the diffuse lobe emission with the imaging capability of XMM-Newton. As seen in Table 4, integration of $1 \mathrm{keV}$ flux densities for E21, E23, E70 and E73 gives $0.7 \mathrm{nJy}$ in total, and hence we can account for a substantial fraction of the difference between the Chandra and XMM-Newton fluxes. Thus we conclude that the Chandra data confirm the detection of non-thermal X-ray emission from the East lobe of 3C 353 and the measurement of its parameters by $\mathrm{G} 08$.

\section{DISCUSSION}

In the previous sections, we have shown that X-ray emission has been detected from the knots of both the jet and the counterjet of the radio galaxy $3 \mathrm{C} 353$. As argued below, this emission is most likely non-thermal in origin. This is in fact the very first clear X-ray detection of an extragalactic counterjet (or, more accurately, of isolated counterjet knots) reported in the literature for a powerful FR II-type source (see the discussion in $§ 1$ ). We have shown (i) that the X-ray knots seem to be narrower than their radio counterparts, (ii) that the Xray-to-radio flux ratio decreases downstream along the jets, and finally (iii) that the intensity maxima of the X-ray jet features are placed closer to the nucleus than the intensity maxima of the nearest radio components (and that these offsets are significant). Moreover, thanks to our deep $90 \mathrm{ks}$ Chandra exposure, the other components of 3C 353 have been successfully detected as well, including the nucleus and the extended East lobe. The nucleus and lobes will briefly be discussed further in $\S 5.1$ and $\S 5.2$ respectively, but since our major findings above are all related to the jets, the remainder of the paper mostly focuses on the interpretation of the observed Xray jet emission. Before this, however, let us comment on the question of whether 3C 353 is indeed representative of other quasar/FR II jet sources.

\subsection{Is It Indeed An FR II Source?}

There are some reasons one might suspect that $3 \mathrm{C} 353$ is not representative of other powerful FR II-type jets. First, 3C 353 is located in the outskirts of a merging cluster, which is not a typical environment for classical doubles or radio loud quasars. Second, one may note the apparent lack of the Xray signatures for the cavity inflated in the cluster medium by the expanding lobes of 3C 353, like those observed in, e.g., Cygnus A (Smith et al. 2002). And third, the particular morphology of the radio hotspots in the discussed object might be thought to be unusual for an FR II radio galaxy: the East (main jet) hotspot is located in the middle - and not at the edge, as expected - of the fat East lobe, while the counterjet terminates in the elongated West lobe without forming a 'classical' hotspot, but instead splits into a mushroom-shaped vortex ring (see Figure 1). This type of jet morphology might rather be thought to resemble the 'inner hotspots', or 'flaring regions' observed in Narrow Angle Tailed (NAT) sources at the point where the substantially curved jets suddenly lose their collimation but continue to propagate away from the nucleus rather than terminating and forming backflow (see in this context Eilek et al. 2002). Interestingly, NAT sources are 
typically (exclusively) located in the outskirts of rich clusters. We therefore considered the possibility that $3 \mathrm{C} 353$ is a NAT radio galaxy observed in projection, with the line of sight being almost in the jet bending plane.

The first reason to doubt the above interpretation is the very low probability that the line of sight would be exactly in the plane in which the NAT jet was bending. With any other orientation of the source in the sky, one should expect to observe some gradual curvature of the 3C 353 outflows, or at least some misalignment between the jet and the counterjet axes. By contrast, the jets in 3C 353 are very straight all over their lengths. As for the jet misalignment, we have carefully investigated the available radio maps, and found only a small difference between the two axes: if a line is drawn from the nucleus out to follow the West jet, and another from the nucleus to follow the East jet, the position angles of the lines differ by $5^{\circ}$. If, instead, we aim for similar size ellipses, one fitting the hotspot region at the end of the counterjet and the other (of the same size) fitting the brightest jet termination feature of the main jet, the lines differ by $1^{\circ}$ only. The other problem is that the jets in NAT sources are of the FR I type, while both the jets in 3C 353 - extremely well collimated outflows with a half-opening angle $\sim 0.5^{\circ}-$ are clear examples of the type of jets seen in FR IIs (Swain 1996, Swain et al. 1998). Also, the apparent lack of any signatures for the X-ray cavity inflated in the ambient medium by the expanding lobes may be explained if 3C 353 is in reality located in front of or behind the cluster mid-plane, as was argued by G08 based on an analysis of pressure balance; even if the source were in the plane of the cluster it is unlikely that a strong cavity signature would be observed at the observed distance from the cluster center. Finally, the recessed hotspot observed in the East lobe of 3C 353 is not particularly unusual for classical doubles (Mullin et al., submitted).

There are also many positive reasons for believing 3C 353 is a fairly typical FR II object, and here we briefly summarize them: (i) the total radio power of $3 \mathrm{C} 353, L_{1.4 \mathrm{GHz}} \sim$ $10^{26} \mathrm{~W} \mathrm{~Hz}^{-1}$, is an order of magnitude above the borderline between FR Is and FR IIs (Fanaroff \& Riley 1974); (ii) the spectral properties of the X-ray nucleus in $3 \mathrm{C} 353$, in particular a large intrinsic absorbing column density $N_{\mathrm{H}}^{G} \sim$ $6 \times 10^{22} \mathrm{~cm}^{-2}$ (see $\S 4.1$ and G08), are typical to what is observed in powerful narrow line radio galaxies, i.e. classical doubles with narrow ionization lines ${ }^{17}$; (iii) the position of $3 \mathrm{C} 353$ in the host (optical) luminosity-radio luminosity domain is consistent with classifying 3C 353 as an FR II source (Swain et al. 1998); (iv) the detection of inverse-Compton $\mathrm{X}$-rays from the lobes of $3 \mathrm{C} 353$ is consistent with what is typically seen in other classical doubles (see $\S 4.3$ and G08). Thus, we conclude that 3C 353 should be considered as representative of the class of powerful jet sources, including both radio-loud quasars and FR II radio galaxies.

\subsection{Origin of the Observed $X$-ray Emission}

Although the constructed three-band X-ray spectra for the jet knots ( $\$ 4.2)$ are consistent with power-law emission, a thermal origin of the detected X-ray features cannot be ruled out by means of spectral analysis, because of the limited photon statistics. In fact, one can fit the X-ray data for these knots

\footnotetext{
${ }^{17}$ However, as noted by G08, the X-ray-to-radio flux ratio of the 3C 353 nucleus is much lower than observed in other narrow line radio galaxies (NL$\mathrm{RGs}$ ), and is more consistent with what is seen in low-excitation radio galaxies (LERGs), so that the nature of 3C 353's nucleus in the X-ray remains ambiguous. Many LERGs exhibit FR II large-scale radio morphology.
}

with a thermal model (bremsstrahlung radiation), although the derived gas temperature is not well constrained. We therefore begin this section by checking whether the global parameters implied by a thermal interpretation are realistic.

The bolometric bremsstrahlung luminosity of a hypothetical source with volume $V$ and number densities of electrons and ions $n_{\mathrm{e}}$ and $n_{\mathrm{i}}$, respectively, assumed to be characterized by a single temperature $T$, is

$$
L_{\mathrm{ff}} \approx 10^{-27} T^{1 / 2} \int n_{\mathrm{e}} n_{\mathrm{i}} d V,
$$

where all the quantities are expressed in cgs units (Blumenthal $\&$ Gould 1970). If we assume $k T \approx 5 \mathrm{keV}$ (which is close to the temperature of the $\mathrm{Zw} 1718.1-0108$ cluster; Iwasawa et al. 2000; G08), and consider a completely ionized thermal hydrogen plasma $\left(n_{\mathrm{e}}=n_{\mathrm{i}}\right)$ uniformly distributed within the spherical volume $V=\pi r^{3}$ of the radius $r \approx 2^{\prime \prime} \approx 1.2 \mathrm{kpc}$ (as appropriate for, e.g., the W47 knot; see Table 4), then we find $L_{\mathrm{ff}} \approx 10^{42} n_{\mathrm{e}}^{2} \mathrm{erg} \mathrm{s}^{-1}$. The observed $0.5-5 \mathrm{keV}$ luminosity of the W47 knot is $L_{\mathrm{X}} \approx 6 \times 10^{39} \mathrm{erg} \mathrm{s}^{-1}$. This, in the framework of the thermal model, would imply a number density of the thermal gas $n_{\mathrm{e}}>0.07 \mathrm{~cm}^{-3}$, which is much higher than what is expected for the thermal gas density at the edge of the cluster. In addition, the corresponding thermal pressure of the hypothetical X-ray emitting cloud, $p_{\text {th }}=n_{\mathrm{e}} k T>6 \times 10^{-10} \mathrm{erg} \mathrm{cm}^{-3}$, would be about two orders of magnitude higher than the non-thermal pressure of the lobe, $p_{\text {lobe }}=U_{\text {lobe }} / 3 \approx 10^{-11} \mathrm{erg} \mathrm{cm}^{-3}$, as estimated below. The presence of such a highly overpressured dense cloud of a hot gas, with a total mass of $M_{\mathrm{tot}}=m_{\mathrm{p}} n_{\mathrm{e}} V>10^{7} M_{\odot}$, within the lobes of a powerful radio galaxy and close to the radio jet, seems highly unlikely. We also emphasize that a clear discontinuity in the polarization properties of the jet, due to the rotation measure associated with the cloud $(R M \approx$ $0.81 n_{\mathrm{e}}\left(B_{\|} / \mu \mathrm{G}\right)(\mathrm{r} / \mathrm{pc}) \mathrm{rad} \mathrm{m}^{-2} \gtrsim 100 \mathrm{rad} \mathrm{m}^{-2}$, for an expected magnetic field component parallel to the line of sight within the cloud $B_{\|} \gtrsim 1 \mu \mathrm{G}$ ), although not large, should have been observable in the detailed analysis of the radio polarization maps presented by Swain (1996) and Swain et al. (1998), but was not seen. Finally, we note that decreasing the assumed gas temperature (in order to reduce the pressure of the postulated X-ray emitting cloud) would cause the derived gas number density, the total mass of the cloud, and the rotation measures to increase still further. Therefore, we rule out a thermal interpretation, and conclude that the X-ray emission of the jet-related knots detected by Chandra is non-thermal in origin.

As can be seen in Figure 12, the overall spectral energy distributions of different knots in 3C 353 are similar to each other, suggesting that the same physical process is responsible for production of the observed X-ray emission from the jet and the counterjet; thus, in what follows, we attempt to find a single emission process that can explain all the observations. In addition, as we have noted above, the X-ray spectra of all the knots are inconsistent with simple extrapolations of the radio (synchrotron) continua. In this situation, as discussed in $\S 1$, the X-ray emission may be synchrotron emission from a second electron population, or it may be some form of inverse-Compton emission. Since the X-ray/radio morphology of the 3C 353 jet knots, discussed in more detail in the next sections (in particular, the large positional offsets between the X-ray and radio intensity maxima, as well as the differences in the X-ray and radio jet widths), exclude any significant contribution of the synchrotron self-Compton 
(SSC) process to the observed X-ray radiative output, we do not consider the SSC process further. Instead we focus our examination of inverse-Compton processes on the IC/CMB model $(\S 1)$. In core-dominated quasars, the spectral behavior seen in the jet and counterjet knots of 3C 353 is often believed to be a manifestation of the IC/CMB component dominating the observed X-ray emission, with corresponding high bulk Lorentz factors (Sambruna et al. 2004, Marshall et al. 2005). We will argue below that this cannot be the case in 3C 353, which substantially weakens the case for adopting the model in analogous FR II/quasar jet sources.

We begin by briefly discussing the global energetics of the 3C 353 jets, by utilizing our Chandra detection of the nonthermal X-ray emission from the East radio lobe (see $\S 4.3$ and ELOBE in Table 1), which is consistent with what was derived from the XMM-Newton data (G08). First, we measure the radio fluxes of the East lobe taking the same extraction region as for the Chandra/XMM-Newton flux measurements, obtaining $89.1 \mathrm{Jy}, 31.7 \mathrm{Jy}, 12.6 \mathrm{Jy}$, and $7.7 \mathrm{Jy}$, respectively for $330 \mathrm{MHz}, 1.4 \mathrm{GHz}, 5 \mathrm{GHz}$, and $8.4 \mathrm{GHz}$ radio maps. This corresponds to a radio spectral index of $\alpha_{\mathrm{R}} \approx 0.75$. Assuming a spherical volume with a radius $R=36 \mathrm{kpc}$ (corresponding to $60^{\prime \prime}$; see $\S 4.3$ ), the equipartition magnetic field strengths for the East lobe is $B_{\text {eq }} \simeq 10 \mu \mathrm{G}$ (e.g., Kataoka $\&$ Stawarz 2005) ${ }^{18}$. By assuming that all the lobe X-ray emission is due to inverse-Comptonization of the cosmic microwave background photons, the measured flux densities imply a magnetic field strength $B_{\text {ic }} \simeq 6 \mu \mathrm{G}$. Therefore, the equipartition magnetic field intensity exceeds the value measured for the East lobe by a factor $\sim 2$. This result is indeed in a very good agreement to what is observed in other FR II sources (see Croston et al. 2005, Kataoka \& Stawarz 2005, and references therein) and consistent with what was measured by G08. Assuming further equal energies stored in the non-thermal electrons and protons, the total energy density of the $3 C 353$ lobes is $U_{\text {lobe }} \sim 3 \times 10^{-11} \mathrm{erg} \mathrm{cm}^{-3}$. The total volume of the lobes is $V_{\text {lobe }} \sim 10^{70} \mathrm{~cm}^{3}$. This gives a total energy $E_{\text {tot }} \sim U_{\text {lobe }} \times V_{\text {lobe }} \sim 3 \times 10^{59} \mathrm{erg}$, which is in fact a lower limit only. Finally, we estimate the radio source lifetime to be $t_{\mathrm{j}} \sim l_{\mathrm{j}} / v_{\mathrm{adv}} \sim 7 \times 10^{6} \mathrm{yrs}$, which corresponds to the observed jet length $l_{\mathrm{j}} \sim 2^{\prime}$ and the assumed advance velocity of the jet termination region $v_{\mathrm{adv}} \sim 0.03 c$, as appropriate for a typical FR II radio galaxy (see, e.g., Machalski et al. 2007). This constrains the jet kinetic power in the 3C 353 radio galaxy to be $L_{\mathrm{j}} \sim E_{\text {tot }} / 2 t_{\mathrm{j}} \sim 10^{45} \mathrm{erg} \mathrm{s}^{-1}$.

The above order-of-magnitude calculations allow us to estimate the jet magnetic field, $B$, by minimizing the total kinetic luminosity of the $3 \mathrm{C} 353$ jets, $L_{\mathrm{j}}=L_{\mathrm{p}}+L_{\mathrm{e}}+L_{\mathrm{B}}$ (the sum of the kinetic powers carried by the jet protons, electrons, and the magnetic field, respectively), for a given jet synchrotron emission (Ghisellini \& Celotti 2001). In particular, with $L_{\mathrm{B}}=\pi R_{\mathrm{j}}^{2} c \Gamma_{\mathrm{j}}^{2} U_{\mathrm{B}}^{\prime}$, where $R_{\mathrm{j}} \approx 1.2 \mathrm{kpc}$ is the jet radius (Swain et al. 1998), $\Gamma_{\mathrm{j}}$ is the jet bulk Lorentz factor, and $U_{\mathrm{B}}^{\prime} \equiv B^{2} / 8 \pi$ is the comoving energy density of the jet magnetic field, one obtains

$$
B=\left(\frac{4 L_{\mathrm{j}}}{R_{\mathrm{j}}^{2} c \Gamma_{\mathrm{j}}^{2}}\right)^{1 / 2} \sim 100 \Gamma_{\mathrm{j}}^{-1} \mu \mathrm{G},
$$

18 See equation (3) in Kataoka \& Stawarz (2005), with minor changes made to mimic the IC/CMB model presented in G08 and Croston et al (2005). In particular, we assume here a broken power-law electron distribution with initial electron energy index $s=2$, and the minimum electron Lorentz factor $\gamma_{\min }=10$ which is very close to the standard equipartition value (Swain 1996). From this, one may find the observer-frame ratio of the IC/CMB and synchrotron luminosities as

$$
\frac{L_{\mathrm{ic} / \mathrm{cmb}}}{L_{\mathrm{syn}}} \approx\left(\frac{\delta_{\mathrm{j}}}{\Gamma_{\mathrm{j}}}\right)^{2} \frac{U_{\mathrm{cmb}}^{\prime}}{U_{\mathrm{B}}^{\prime}} \sim 10^{-3} .
$$

Here, $U_{\mathrm{cmb}}^{\prime} \approx 4 \times 10^{-13} \Gamma_{\mathrm{j}}^{2} \mathrm{erg} \mathrm{cm}^{-3}$ is the jet comoving energy density of the $\mathrm{CMB}$ radiation, $U_{\mathrm{B}}^{\prime} \sim$ $4 \times 10^{-10} \Gamma_{\mathrm{j}}^{-2} \mathrm{erg} \mathrm{cm}^{-3}$ (see equation 2 ), and $\delta_{\mathrm{j}} \equiv$ $\left[\Gamma_{\mathrm{j}}\left(1-\beta_{\mathrm{j}} \cos \theta_{\mathrm{j}}\right)\right]^{-1}$ is the jet Doppler factor.

Based on their models of the jet total intensity and polarization profiles, Swain et al. (1998) constrained the jet inclination to the line of sight in $3 \mathrm{C} 353$ to be $60^{\circ}<\theta_{\mathrm{j}}<90^{\circ}$; these large angles to the line of sight are strongly supported by the observed two-sidedness of both the radio and X-ray jets. Accordingly, we can approximately substitute $\delta_{\mathrm{j}} \sim 1 / \Gamma_{\mathrm{j}}$ in equation 3, obtaining the observed luminosity ratio $L_{\mathrm{ic} / \mathrm{cmb}} / L_{\mathrm{syn}}$ independently of the jet kinematic factors. The resulting value, $\sim 10^{-3}$, is in strong disagreement with the observed X-ray-to-radio luminosity ratio $L_{\mathrm{X}} / L_{\mathrm{R}} \gtrsim 1$ (see Figure 12 ). Note, that in the framework of the IC/CMB model with large jet inclinations such that $\delta_{\mathrm{j}} \sim 1 / \Gamma_{\mathrm{j}}$, the electrons producing inverse-Compton emission at the observed $\mathrm{keV}$ photon energies have Lorentz factors $\gamma_{\mathrm{X}} \sim\left(\nu_{\mathrm{keV}} / \nu_{\mathrm{cmb}} \delta_{\mathrm{j}}^{2}\right)^{1 / 2} \sim$ $10^{3} \Gamma_{\mathrm{j}}$, i.e., almost exactly the same as the electrons producing synchrotron photons at the observed $\mathrm{GHz}$ frequencies, $\gamma_{\mathrm{R}} \sim\left(\nu_{\mathrm{GHz}} / 4.2 \times 10^{6} B \delta_{\mathrm{j}}\right)^{1 / 2} \sim 10^{3} \Gamma_{\mathrm{j}}$, so one can write $L_{\mathrm{ic} / \mathrm{cmb}} / L_{\mathrm{syn}} \sim L_{\mathrm{X}} / L_{\mathrm{R}}$. Thus, we conclude that the IC/CMB model cannot explain the observed X-ray emission of the 3C 353 jets, unless very large departures from energy equipartition, $B \ll 100 \Gamma_{\mathrm{j}}^{-1} \mu \mathrm{G}$, are invoked. Such large deviations from the minimum power condition are not expected in the case of large-scale extragalactic jets (see in this context Stawarz et al. 2005, 2006) and they would imply very high total energy densities in the jet.

The only possibility left is therefore that the observed Xray emission of 3C 353 results from the synchrotron radiation of some flat-spectrum high-energy electron population, most likely separate to the one producing the observed radio emission. The required Lorentz factors of the electrons emitting synchrotron photons with the observed $\mathrm{keV}$ energies are $\gamma_{\mathrm{X}} \sim\left(\nu_{\mathrm{keV}} / 4.2 \times 10^{6} B \delta_{\mathrm{j}}\right)^{1 / 2} \sim 3 \times 10^{7} \Gamma_{\mathrm{j}}$ (assuming the scaling of the magnetic field as given in equation 2 above and, again, $\delta_{\mathrm{j}} \sim 1 / \Gamma_{\mathrm{j}}$ ). It has already been shown that stochastic acceleration processes taking place in large-scale extragalactic outflows may easily account for the production of electrons with these Lorentz factors (Stawarz \& Ostrowski 2002, Uchiyama et al. 2006) and they are already invoked to explain observations of the hotspots of FR II sources and the jets of FR Is $(\S 1)$. In the following section, by discussing in detail the X-ray/radio morphology of the 3C 353 jets, we aim to constrain several other aspects of the synchrotron scenario.

\subsection{Radio/X-ray Jet Morphology}

Before discussing in more details the radio/X-ray morphology of the $3 C 353$ jets, it is useful to summarize the main results of the extensive radio studies carried out on this source by Swain (1996) and Swain et al. (1998). By means of detailed modeling of the intensity and polarization properties of the jets in $3 \mathrm{C} 353$, these authors concluded that:

- most of the observed radio emission from the jet (as well as from the counterjet), is produced within the 
outer sheath of the outflow/jet boundary layer with a velocity shear; the contribution from the central jet spine is negligible;

- the magnetic field within the jet boundary layer has no radial component, $B_{r}=0$, but only random axial and toroidal components in equipartition, $\left\langle B_{z}^{2}\right\rangle^{1 / 2} \approx$ $\left\langle B_{\phi}^{2}\right\rangle^{1 / 2}$, both ordered by a velocity shear; the axial component of the magnetic field within the jet spine is negligible;

- the outer sheath and the jet spine have constant halfopening angle $\Theta \approx 0.5^{\circ}$ all along the outflow, such that the radius of the spine is roughly half of the total jet radius $R_{\mathrm{j}} \approx 2^{\prime \prime} \approx 1.2 \mathrm{kpc}$;

- jet models with ordered large-scale magnetic field (helical structure, transverse flux ropes, etc.) cannot account for the observed flat-topped synchrotron intensity profiles across the jets and, at the same time, for the symmetric polarization profiles with measured degree of linear polarization $\sim 10 \%-20 \%$.

\subsubsection{The Widths of the Radio and X-ray Jets}

As described in $\$ 3.2$ of this paper, we have found that the X-ray knots in 3C 353 are possibly narrower than their radio counterparts (which are also situated further away from the active nucleus). This agrees with what is observed in powerful quasar jets detected by Chandra: the limits to the width of the (transversely unresolved) X-ray knots in these systems are always slightly smaller than the measured sizes in the radio band, and sometimes even in the optical band (Jester et al. $2006,2007)$. In the framework of the IC/CMB model involving significant beaming, one would argue that since the comoving energy density of the CMB photons is proportional to the square of the bulk Lorentz factor of the emitting plasma, $U_{\mathrm{cmb}}^{\prime} \approx \Gamma_{\mathrm{j}}^{2} U_{\mathrm{cmb}}$, the comoving IC/CMB emissivity of the slower jet boundary layer should be much smaller than the analogous emissivity of a fast jet spine, resulting in the $\mathrm{X}$ ray jet being narrower than its radio/optical counterpart (for a fixed magnetic field energy density $U_{\mathrm{B}}^{\prime}$ throughout the outflow). However, as discussed in the previous section, the detection of the X-ray counter-knot in 3C 353 and the established large inclination of this source exclude the IC/CMB model: only the synchrotron model is viable to explain these features. Thus, the observations imply that the presence of high-energy electrons producing the observed synchrotron $\mathrm{X}$ ray jet emission must be restricted to the central spine of the jet rather than to the jet boundary layer, at least in the upstream portions of the bright knots, if our findings regarding the widths of the X-ray jet features are correct. We speculate that such a difference may be due to the different configurations of the small-scale magnetic field within different parts of the radially stratified outflow, as discussed by Swain et al. (1998).

It should be borne in mind, however, that with the current data the signal-to-noise ratio of the Chandra X-ray maps of 3 C 353 is rather poor, and therefore all conclusions regarding the width of the X-ray jets in this object, or rather of the isolated jet knots, should be taken with extreme caution. Also, we emphasize that the multiwavelength structures and spectra of bright knots in other well-studied jet sources (3C 273, Centaurus A) do not necessarily correspond directly to the multiwavelength morphology and spectral properties of the interknot regions (Marshall et al. 2005b, Kataoka et al. 2006, Hardcastle et al. 2007b), but that it is the interknot regions (rather than the bright knots) that reflect closely the global, possibly stratified morphology of the outflow. Finally, the poorly known internal structure of the boundary layer in relativistic jets may be much more complicated than what is typically assumed, resulting in several non-intuitive radiative properties of the jet plasma (see in this context Aloy \& Mimica 2008).

\subsubsection{Decrease of the $F_{\mathrm{X}} / F_{\mathrm{R}}$ Flux Ratio Along the Jets}

In the case of the jets in $3 \mathrm{C} 353$, we observe a systematic decrease of the $F_{\mathrm{X}} / F_{\mathrm{R}}$ ratio along the outflow (see $\S 4.2$ and Figure 11). Similar behavior is observed in many (though not all) quasar jets detected by Chandra (e.g., Sambruna et al. 2004, Marshall et al. 2005a, Hardcastle 2006, Siemiginowska et al. 2007). In the framework of the IC/CMB model for the $\mathrm{X}$-ray jet emission, it has been suggested that this behavior results from a smooth deceleration of the outflows on kpc-Mpc scales due to gradual mass entrainment (Georganopoulos \& Kazanas 2004, Tavecchio et al. 2006). As pointed out by Hardcastle (2006), this idea faces several difficulties in explaining the multiwavelength morphology of powerful jets, and here we briefly summarize the arguments given: (i) no trend for the gradual bulk deceleration is observed in largescale radio jets of FR II sources, but if only the jet spine is to be decelerated, the model should explain why the jet boundaries do not respond to the involved dramatic changes in the jet/spine kinematics; (ii) the amount of cold matter required to be entrained, and thus to decelerate powerful quasar jets, is in some cases too high when compared to the upper limits on the masses of the surrounding thermal gas. As discussed previously, the IC/CMB model is excluded in the case of $3 \mathrm{C} 353$. On the other hand, in the previous subsection we have argued that the X-ray morphology implies that the synchrotron Xray emission is associated with the faster jet spine rather than with the slower jet boundaries. Could the observed systematic decrease of the $F_{\mathrm{X}} / F_{\mathrm{R}}$ flux ratio therefore nevertheless be connected with a smooth jet/spine deceleration?

It is important to remark again in this context that the jets in 3C 353 are extremely well collimated all over their lengths, with a constant half-opening angle $\Theta \approx 0.5^{\circ}$, and constant jet/counterjet brightness asymmetry ratio $\mathcal{R}_{\mathrm{R}} \approx 2$. This is in agreement with what is observed in other FR IIs: no evidence for gradual jet deceleration is provided by the jet radio morphology (Hardcastle 2006). Moreover, as pointed out by Swain et al. (1998), the fact that the boundary layer seems to be always restricted to the same parts of the outflow $>0.5 R_{\mathrm{j}}$ (i.e., that the boundary shear layer does not spread/expand toward the jet axis), coupled with the constancy of the observed level of linear polarization along the jets and the absence of radial component of the magnetic field, indicate that no largescale turbulent eddies associated with mass entrainment are present in the 3C 353 jets. Since it is the jet boundary shear layer which mediates interaction of the jet with the surrounding medium, the absence of changes in the shear layer implies that there is no reason for the jet spine to decelerate. Thus, we must conclude that systematic mass entrainment on scales of tens of kpc is unlikely to be of any importance, at least in $3 \mathrm{C} 353$. The only remaining possibility is thus that the particle acceleration conditions associated with microphysical plasma parameters - rather than with the global hydrodynamical configuration of the outflow - change within the central parts of the jets. Because those regions of the jet dom- 
inate the jet radiative output at X-ray frequencies but produce negligible radio emission when compared to the jet boundaries, the ratio of the observed X-ray and radio fluxes decreases systematically along the jet in 3C 353 and in other analogous systems. We might for example speculate that a gradually decaying chaotic component of the magnetic field in the jet spine leads to a lower efficiency in the acceleration (due to, e.g., magnetic reconnection processes) of the highestenergy electrons.

\subsubsection{Positional Offsets Between the X-ray and Radio Knots}

The apparent positional offsets between the intensity maxima of the X-ray and radio knots, as reported in this paper for 3C 353, are observed in several other jet sources detected by Chandra (see, e.g., Siemiginowska et al. 2007). As discussed by Hardcastle et al. (2003), they cannot be explained by simple models involving particle acceleration at a single extended shock front, which just exploit the difference between the cooling timescales for the electrons emitting synchrotron Xray and radio photons (Bai \& Lee 2003). In a wider context, the problem of such offsets relates directly to the question on the nature of the jet knots: are they separate moving portions of the jet matter, or rather stationary features produced by, e.g., reconfinement shocks? We note that formation of reconfinement shocks in relativistic outflows has recently been widely discussed in the context of the inner $(<1 \mathrm{kpc})$ jets of $\gamma$-ray emitting blazars and radio galaxies (e.g., Jorstad et al. 2001, Cheung et al. 2007, Levinson \& Bromberg 2008). Reconfinement shocks have been proposed to explain the global morphology of kpc-scale FR I jets (Laing \& Bridle 2002), and also - which is the most interesting in the context of our discussion - the almost regularly spaced knots in FR II jets on scales of tens to hundreds of kpc (Komissarov 1994, Komissarov \& Falle 1998). On the other hand, the frequency independence of the longitudinal profiles of the knots in the quasar jets observed by Chandra, among other observed jet properties, has led several authors to conclude that knots are instead separate moving portions of the jet with excess kinetic power, produced by intermittent or modulated activity of the central engine (Bridle et al. 1986, 1989, Clarke et al. 1992, Stawarz et al. 2004).

The results of the analysis of the radio data for the 3C 353 jets presented by Swain et al. (1998), in particular the reported constant opening angle of both the jet spine and the jet boundary layer, is hard to reconcile with the expected 'diamond structure' of the successive reconfinement shocks discussed by Komissarov \& Falle (1998). In addition, the reconfinement shock scenario does not predict in a natural way the frequency-dependent position of the intensity maxima of the radiating plasma. Clearly, this problem cannot be addressed fully in our qualitative discussion, since detailed numerical simulations are needed to understand the complex structure of the reconfinement shocks in large-scale mildly-relativistic outflows. However, we believe that a more likely explanation for the observed X-ray/radio positional offsets can be given in the framework of the model in which knots are moving portions of the jet matter. For example, one may propose that portions of the jet produced by the central engine during a more active period, and hence characterized by excess kinetic power and/or higher bulk velocity, propagate within the 'stationary' (slower) outflow corresponding to the quasiquiescent state of the active nucleus. Since the bulk velocities of the jet plasma are expected to be supersonic (although not necessarily ultrarelativistic), a double-shock structure is ex- pected to form, due to collisions of the faster portions of the jet matter with the slower outflow.

Let us consider a model in which the forward shock propagating in the slower outflow (and thus characterized by a highly oblique geometry at the jet boundaries) is responsible for the brightening of the outer portions of the jet at radio frequencies (due to compression of the jet magnetic field, driving the turbulence, etc.), while the reverse shock propagating within the faster portion of the jet is associated with the intensity maximum of the synchrotron X-ray emission. In this case we may make some simple illustrative calculations of the expected magnitude of the offsets. Let $\Gamma_{1}$ be the bulk Lorentz factor of the slower (upstream) portion of the outflow, and $\Gamma_{2}$ be the bulk Lorentz factor of the faster (but otherwise identical) portion of the jet. After the collision, a symmetric double-shock structure forms within the outflow, for which the contact discontinuity propagates with the velocity $\beta_{\mathrm{j}}=\left(1-\Gamma_{\mathrm{sh}}^{-2}\right)^{1 / 2}$ in the observer's frame. This is, of course, the bulk velocity of the radiating (downstream) jet plasma. Let us also assume that the slow portion of the outflow is at most mildly relativistic (see the discussion in the previous sections), i.e., that $\Gamma_{1} \sim 1$. With this assumption, the velocity of the shock in the observer frame is $\beta_{\mathrm{sh}}=\left(1-\Gamma_{\mathrm{sh}}^{-2}\right)^{1 / 2}$, where

$$
\Gamma_{\mathrm{sh}}^{2}=\frac{\left(\Gamma_{\mathrm{j}}+1\right)\left[\hat{\gamma}\left(\Gamma_{\mathrm{j}}-1\right)+1\right]^{2}}{\hat{\gamma}(2-\hat{\gamma})\left(\Gamma_{\mathrm{j}}-1\right)+2},
$$

and $\hat{\gamma}$ is the ratio of the specific heats of the jet plasma (see, e.g., Stawarz et al. 2004, Appendix E). The extent of the shocked region in the downstream plasma rest frame (denoted by primes) is $\Delta l^{\prime}=2 c \beta_{\mathrm{sh}}^{\prime} \Delta t^{\prime}$, where $\beta_{\mathrm{sh}}^{\prime}=\left(\beta_{\mathrm{sh}}-\beta_{\mathrm{j}}\right) /(1-$ $\left.\beta_{\mathrm{sh}} \beta_{\mathrm{j}}\right)$, and $\Delta t^{\prime}$ is the comoving time since collision. Because for the moving source $\Delta l=\delta_{\mathrm{j}} \Delta l^{\prime}$ and $\Delta t=\Gamma_{\mathrm{j}} \Delta t^{\prime}$, we obtain the expected separation of the radio and X-ray intensity maxima (i.e., the separation between the forward and reverse shock)

$$
\Delta l=2 c \beta_{\mathrm{sh}}^{\prime} \Gamma_{\mathrm{j}}^{-2} \Delta t,
$$

where we take $\delta_{\mathrm{j}} \equiv 1 / \Gamma_{\mathrm{j}}\left(1-\beta_{\mathrm{j}} \cos \theta_{\mathrm{j}}\right) \sim 1 / \Gamma_{\mathrm{j}}$ as appropriate for a jet viewed at large inclinations $\theta_{\mathrm{j}} \lesssim 90^{\circ}$. Note that in the framework of the proposed toy model, one should expect the spatial offset $\Delta l$ to increase with $\Delta t$, that is, with the distance from the nucleus. This is in agreement with what is observed in 3 C 353 . Note also that the timescale required to explain the kpc-scale positional differences between the intensity maxima of the knots, $\Delta t \approx 3 \times 10^{4}(\Delta l / \mathrm{kpc}) \mathrm{yrs}$, is in a very good agreement with what has been proposed for the intermittent activity of the central engine in powerful FR II/quasar sources (Reynolds \& Begelman 1997, Siemiginowska \& Elvis 1997, Stawarz et al. 2004). In the above estimate we have assumed a very moderate bulk Lorentz factor for the jet $\Gamma_{\mathrm{j}}=2$ (corresponding to a bulk velocity of the radiating plasma $\beta_{\mathrm{j}}=0.866$ ), and the ratio of the specific heats $\hat{\gamma}=4 / 3$. For these parameters we find $\Gamma_{\mathrm{sh}}=2.38$, $\beta_{\mathrm{sh}}=0.907$, and $\beta_{\mathrm{sh}}^{\prime}=0.193$.

Several features of the observations are not explained by this scenario; for example, (i) there is no obvious reason why the forward and reverse fronts of the double shock structure should work substantially differently with respect to the acceleration of ultrarelativistic electrons; and (ii) it is not clear why similar positional offsets are observed in the jet knots and in the jet termination regions (hotspots), since the shock structures produced in the latter regions are expected to be completely different. These may suggest that the toy model considered above is over-simplified, and that some other physical 
picture has to be considered. Another possibility — still in the framework of intermittent jet activity - is that the central engine ejects heavy knots in $\sim 10^{4}$ yr-long flare-like activity periods. Due to the high (relativistic) mass load of the knot, the bulk velocity of one of these heavier portions of magnetized plasma is expected to be smaller than the velocity of the "weak jet' formed in an epoch of quiescence. Interactions between the heavy/slower and light/faster portions of the jet would lead to formation of a single (reverse) shock at the upstream edge of a heavy knot (the forward shock, propagating within the heavy portion of the jet matter, would be expected to die away soon after its formation, as long as the density ratio between the two phases of the ejecta was high). Thus, the heavy portion of the jet outflow would behave like the reflecting walls used in 'particle-in-cell' simulations and would form a shock front that would propagate upstream. As a result, the positions of radio knots would coincide with the positions of heavy portions of the jet plasma, while the X-ray intensity maxima due to freshly accelerated electrons would be expected to coincide with the downstream region of the reverse shock. This model solves at least problem (i) noted above; however, discussions based on more sophisticated numerical simulations are clearly essential to confirm this or other similar speculations.

\section{CONCLUSIONS}

We have presented a detailed analysis of the data for the powerful FR II radio galaxy 3C 353 obtained with the Chandra X-ray Observatory. In a deep, $90 \mathrm{ks}$ Chandra observation, we have detected the X-ray emission from most of the radio structures in this source, including the nucleus, isolated knots in the jet and the counterjet, the terminal jet regions (hotspots), and one radio lobe. Our major findings are as follows:

(i). Non-thermal X-ray emission associated with a knot in the counterjet of a powerful FR II source has been detected for the first time. This detection, in agreement with the established large inclination of 3C 353, strongly disagrees with the inverse-Compton model proposed in the literature, and points to a synchrotron origin for the X-ray jet photons.

(ii). We find that the width of the X-ray knots is narrower ( $\gtrsim 4 \sigma$ effect) than that measured at radio wavelengths, suggesting that the production of the X-ray emission is associated with the central jet spine rather than with the jet boundaries. This conclusion, however, must be confirmed with X-ray data with a much better signalto-noise ratio.

(iii). The radio-to-X-ray flux ratio decreases systematically downstream along the outflows, as is often observed in other extragalactic sources. We argue that this is due to the fact that the particle acceleration conditions associated with microphysical plasma parameters changes within the central part of the jet, rather than being due to a gradual decrease of the jet bulk velocity resulting from a smooth jet deceleration.

(iv). Substantial (kpc-scale) offsets between positions of the $\mathrm{X}$-ray and radio intensity maxima within each knot are found, with the magnitude of the offsets increasing away from the nucleus. We have speculated that these offsets can be explained if radio knots are moving portions of jet material which are produced by the central engine during an epoch of enhanced activity. Depending on the velocity and density ratio between these knots and the outflow produced at times of quiescence, a complex double-shock structure may form as a result of the interaction between the two phases of the ejecta, with the reverse shock (propagating within the faster portion of the jet) being associated with the peak of the $\mathrm{X}$-ray emission.

Although we cannot provide definitive solutions or interpretations for each problem, we argue that the 3C 353 data strongly suggest that the synchrotron X-ray emission of extragalactic large-scale jets is not simply shaped by the global hydrodynamical configuration of the outflows, but is also very sensitive to the microphysical parameters of the jet plasma.

J.K. acknowledges support by JSPS KAKENHI (19204017/14GS0211). Ł.S. and M.O. acknowledge support by the MEiN grant 1-P03D-003-29. M.J.H. acknowledges support from the Royal Society and J.L.G. thanks the UK Science and Technology Facilities Council for a studentship. M.O. thanks Malgosia Mochol for her assistance in the evaluation of the Chandra data. This research is funded in part by NASA contract NAS8-39073. Partial support for this work was provided by the National Aeronautics and Space Administration through Chandra Awards Number GO5-6113X and GO7-8103X-R issued by the Chandra X-Ray Observatory Center, which is operated by the Smithsonian Astrophysical Observatory for and on behalf of NASA under contract NAS8-39073. This research has made use of data obtained by Chandra X-ray Observatory and software provided by the Chandra X-ray Center (CXC). This research has also made use of SAOImage DS9, developed by Smithsonian Astrophysical Observatory. The National Radio Astronomy Observatory is a facility of the National Science Foundation operated under cooperative agreement by Associated Universities, Inc.

\section{REFERENCES}

Aloy, M.A., \& Rezzolla, L., 2006, ApJ, 640, L115

Aloy, M.A., \& Mimica, P., 2008, ApJ, in press (arXiv:0803.2693)

Aloy, M.A., Ibanez, J.M., Martì, J.M., Gómez, J.-L., \& Müller, E., 1999, ApJ, 523, L125

Bai, J.M., \& Lees, M.G., 2003, ApJ, 585, L113

Begelman, M.C., Blandford, R.D., \& Rees, M.J., 1984, Rev.Mod.Phys., 56, 255

Blumenthal, G.R., \& Gould, R.J., 1970, Rev.Mod.Phys., 42, 237

Bridle, A.H., \& Perley, R.A., 1984, ARA\&A, 22, 319

Bridle, A.H., Perley, R.A., \& Henriksen, R.N., 1986, AJ, 92, 534

Bridle, A.H., Fomalont, E.B., Byrd, G.G., \& Valtonen, M.J., 1989, AJ, 97, 674
Carilli, C.L., Harris, D.E., Pentericci, L., Rottgering, H.J.A., Miley, G.K., Kurk, J.D., \& van Breugel, W., 2002, ApJ, 567, 781

Celotti, A., Ghisellini, G., \& Chiaberge, M., 2001, MNRAS, 321, L1

Cheung, C.C., Harris, D.E., \& Stawarz, Ł., 2007, ApJL, 663, 65

Chiaberge, M., Gilli, R., Macchetto, F.D., Sparks, W.B., \& Capetti, A., 2003, ApJ, 582, 645

Clarke, D.A., Bridle, A.H., Burns, J.O., Perley, R.A., \& Norman, M.L., 1992, ApJ, 285, 173

Croston, J.H., Hardcastle, M.J., Harris, D.E., Belsole, E., Birkinshaw, M., \& Worrall, D.M., 2005, ApJ, 626, 733

Dickey, J.M., \& Lockman, F.J., ARA\&A, 28, 215 
Eilek, J.A., Hardee, P.E., Markovic, T., Ledlow, M., \& Owen, F.N., 2002, NewAR, 46, 327

Evans, D.A., Worrall, D.M., Hardcastle, M.J., Kraft, R.P., \& Birkinshaw, M., 2006, ApJ, 642, 96

Fomalont, E.B., Frey,S., Paragi, Z., Gurvits, L. I., Scott, W. K., Taylor, A. R., Edwards, P. G., \& Hirabayashi, H. 2000, ApJS, 131, 95

Fan, Z.-H., Liu, S., Wang, J.-M., Fryer, C.L., \& Li, H., 2008, ApJL, 673, L139

Fanaroff, B.L., \& Riley, J.M., 1974, MNRAS, 167, 31

Georganopoulos, M., \& Kazanas, D., 2004, ApJ, 604, 81

Ghisellini, G., \& Celotti, A., 2001, MNRAS, 327, 739

Goodger, J.L., Hardcastle, M.J., Croston, J.H., Kassim, N.E., \& Perley, R.A., 2008, MNRAS, 386, 337 [G08]

Hardcastle, M.J., et al., 1999, MNRAS, 304, 135

Hardcastle, M.J., Worrall, D.M., Kraft, R.P., Forman, W.P., Jones, C., \& Murray, S.S., 2003, ApJ, 593, 169

Hardcastle, M.J., Harris, D.E., Worrall, D.M., \& Birkinshaw, M., 2004, ApJ, 612,729

Hardcastle, M.J., \& Croston, J.H., 2005, MNRAS, 363, 649

Hardcastle, M.J., 2006, MNRAS, 366, 1465

Hardcastle, M.J., Evans, D. A., \& Croston, J. H., 2006, MNRAS, 370, 1893

Hardcastle, M.J., Croston, J.H., \& Kraft, R.P., 2007a, ApJ, 669, 893

Hardcastle, M.J., et al. 2007b, ApJL, 670, L81

Hardee, P.E., 2007, ApJ, 664, 26

Harris, D.E., \& Krawczynski, H., 2002, ApJ, 565, 244

Harris, D.E., \& Krawczynski, H., 2006, ARA\&A, 44, 463

Honda, M., \& Honda, Y.S., 2007, ApJ, 654, 885

Iwasawa, K., Ettori, S., Fabian, A.C., Edge, A.C., \& Ebeling, H., 2000, MNRAS, 313, 515

Jamrozy, M., Konar, C., Saikia, D.J., Stawarz, Ł., Mack, K.-H., \& Siemiginowska, A., 2007, MNRAS, 378, 581

Jester, S., Röser, H.-J., Meisenheimer, K., \& Perley, R. 2005, A\&A, 431, 477

Jester, S., Harris, D.E., Marshall, H.L., \& Meisenheimer, K., 2006, ApJ, 648, 900

Jester, S., Meisenheimer, K., Martel, A.R., Perlman, E.S., \& Sparks, W.B., 2007, MNRAS, 380, 828

Jorstad, S.G., Marscher, A.P., Mattox, J.R., Wehrle, A.E., Bloom, S.D., \& Yurchenko, A.V., 2001, ApJS, 134, 181

Kataoka, J., Edwards, P., Georganopoulos, M., Takahara, F., \& Wagner, S., 2003a, A\&A, 399, 91

Kataoka, J., Leahy, J. P., Edwards, P.G., Kino, M., Takahara, F., Serino, Y., Kawai, N., \& Martel, A.R., 2003b, A\&A, 410, 833

Kataoka, J., \& Stawarz, Ł., 2005, ApJ, 622, 797

Kataoka, J., Stawarz, Ł., Aharonian, F., Takahara, F., Ostrowski, M., \& Edwards, P.G., 2006, ApJ, 641, 158

Komissarov, S.S., 1994, MNRAS, 266, 649

Komissarov, S.S., \& Falle, S.A.E.G., 1998, MNRAS, 297, 1087

Kraft, R.P., Hardcastle, M.J., Worrall, D.M., \& Murray, S.S., 2005, ApJ, 622, 149

Laing, R.A., \& Bridle, A.H. 2002, MNRAS, 336, 1161

Laing, R.A., \& Bridle, A.H., 2004, MNRAS, 348, 1459

Laing, R.A., Canvin, J.R., Cotton, W.D., \& Bridle, A.H., 2006a, MNRAS, 368,48

Laing, R.A., Canvin, J.R., Bridle, A.H., \& Hardcastle, M.J., 2006b, MNRAS, 372,510

Leismann, T., Antòn, L., Aloy, M.A., Müller, E., Martí, J.M., Miralles, J.A.; \& Ibánez, J.M., 2005, A\&A, 436, 503

Levinson, A., \& Bromberg, O., 2008, in Proc. "High Energy Phenomena in Relativistic Outflow", Dublin, Ireland, September 24-28, 2007 (astro$\mathrm{ph} / 0712.2664 \mathrm{v} 1)$

Machalski, J., Chyży, K.T., Stawarz, Ł., \& Kozieł, D., 2007, A\&A, 462, 43

Marshall, H. L., et al., 2001, ApJ, 549, L167

Marshall, H.L., Miller, B.P., Davis, D.S., Perlman, E.S., Wise, M., Canizares, C.R., \& Harris, D.E., 2002, ApJ, 564, 683
Marshall, H.L., Schwartz, D.A., Lovell, J.E.J., Murphy, D.W., Worrall, D.M., Birkinshaw, M., Gelbord, J.M., Perlman, E.S., \& Jauncey, D.L., 2005a, ApJS, 156, 1

Marshall, H.L., Jester, S., Harris, D.E., \& Meisenheimer, K., 2005b, in Proc. 'The X-ray Universe 2005', San Lorenzo de El Escorial, Spain, 26-30 September 2005 (astro-ph/0511145)

Meliani, Z., \& Keppens, R., 2007, A\&A, 475, 785

Mizuno, Y., Hardee, P., \& Nishikawa, K.-I., 2007, ApJ, 662, 835

Mizuno, Y., Hardee, P., Hartmann, D.H., Nishikawa, K.-I., \& Zhang, B. 2008, ApJ, 672, 72

Monet, D., et al. 2003, AJ, 125, 984

Mullin, L.M., Riley, J.M., \& Hardcastle, M.J., 2008, MNRAS, submitted

Perucho, M., Hanasz, M., Martí, J.-M., \& Miralles, J.-A., 2007, PhRvE, 75, 6312

Reynolds, C.S., \& Begelman, M.C., 1997, ApJ, 487, 135

Sambruna, R.M., Gambill, J.K., Maraschi, L., Tavecchio, F., Cerutti, R., Cheung, C.C., Urry, C.M., \& Chartas, G., 2004, ApJ, 608, 698

Sambruna, R.M., Gliozzi, M., Tavecchio, F., Maraschi, L., \& Foschini, L., 2006, ApJ, 652, 146

Sambruna, R.M., Donato, D., Tavecchio, F., Maraschi, L., Cheung, C.C., \& Urry, C.M., 2007, ApJ, 670, 74

Scarpa, R., \& Urry, C.M., 2002, NewAR, 46, 405

Schlegel, D.J., Finkbeiner, D.P. \& Davis, M. 1998, ApJ, 500, 525

Schwartz, D.A., et al., 2000, ApJ, 540, L69

Siemiginowska, A., \& Elvis, M., 1997, ApJ, 482, 9

Siemiginowska, A., Bechtold, J., Aldcroft, T.L., Elvis, M., Harris, D.E., Dobrzycki, A., 2002, ApJ, 570, 543

Siemiginowska, A., Stawarz, Ł., Cheung, C.C., Harris, D.E., Sikora, M., Aldcroft, T.L., \& Bechtold, J., 2007, ApJ, 657, 145

Smith, D.A., Wilson, A.S., Arnaud, K.A., Terashima, Y., \& Young, A.J., 2002, ApJ, 565, 195

Stawarz, Ł., 2003, ChJAS, 3, 383

Stawarz, Ł., \& Ostrowski, M., 2002, ApJ, 578, 763

Stawarz, Ł., Sikora, M., Ostrowski, M., \& Begelman, M.C., 2004, ApJ, 608, 95

Stawarz, Ł., Siemiginowska, A., Ostrowski, M., Sikora, M., 2005, ApJ, 626, 120

Stawarz, Ł., Kneiske, T. M., \& Kataoka, J., 2006, ApJ, 637, 693

Stawarz, Ł., Cheung, C.C., Harris, D.E., \& Ostrowski, M., 2007, ApJ, 662, 213

Steenbrugge, K.C., Blundell, K.M., \& Duffy, P., 2008, in Proc. "High Energy Phenomena in Relativistic Outflows", Dublin, Ireland, September 24-28, 2007 (astro-ph/0712.3024v1)

Swain, M.R., 1996, Ph.D. Thesis, University Rochester

Swain, M.R., Bridle, A.H., \& Baum, S.A., 1998, ApJL, 507, L29

Tavecchio, F., Maraschi, L., Sambruna, R.M., \& Urry, C.M., 2000, ApJL, 544, L23

Tavecchio, F., Maraschi, L., Sambruna, R.M., Urry, C.M., Cheung, C.C., Gambill, J.K., \& Scarpa, R., 2004, ApJ, 614, 64

Tavecchio, F., Cerutti, R., Maraschi, L., Sambruna, R.M., Gambill, J.K., Cheung, C.C., \& Urry, C.M., 2005, ApJ, 630, 721

Tavecchio, F., Maraschi, L., Sambruna, R.M., Gliozzi, M., Cheung, C.C., Wardle, J.F.C., \& Urry, C.M., 2006, ApJ, 641, 732

Tavecchio, F., Maraschi, L., Wolter, A., Cheung, C.C., Sambruna, R.M., \& Urry, C.M., 2007, ApJ, 662, 900

Uchiyama, Y., et al., 2006, ApJ, 648, 910

Wardle, J.F.C., \& Aaron, S.E., 1997, MNRAS, 286, 425

Worrall, D.M., et al., 2008, ApJ, 673, L135

van Breugel, W.J.M., Heckman, T.M., Miley, G.K., \& Filippenko, A.V., 1986, ApJ, 311, 58

Vikhlinin, A., Forman, W., Jones, C., \& Murray, S. 1995, ApJ, 451, 542 